\title{
Chronic linaclotide treatment reduces colitis-induced neuroplasticity and reverses persistent bladder dysfunction
}

\author{
Luke Grundy, ${ }^{1,2}$ Andrea M. Harrington, ${ }^{1,2}$ Joel Castro, ${ }^{1,2}$ Sonia Garcia-Caraballo, ${ }^{1,2}$ Annemie Deiteren, ${ }^{1,2}$ \\ Jessica Maddern, ${ }^{1,2}$ Grigori Y. Rychkov, ${ }^{2}$ Pei Ge, ${ }^{3}$ Stefanie Peters, ${ }^{4}$ Robert Feil, ${ }^{4}$ Paul Miller, ${ }^{5}$ \\ Andre Chetti, ${ }^{5}$ Gerhard Hannig, ${ }^{3}$ Caroline B. Kurtz, ${ }^{6}$ Inmaculada Silos-Santiago, ${ }^{6}$ \\ and Stuart M. Brierley ${ }^{1,2}$ \\ 'Visceral Pain Research Group, Centre for Neuroscience, College of Medicine and Public Health, Flinders University, Bedford \\ Park, South Australia, Australia. ${ }^{2}$ Centre for Nutrition and Gastrointestinal Diseases, Discipline of Medicine, University of \\ Adelaide, North Terrace, Adelaide, South Australia, Australia, and South Australian Health and Medical Research Institute \\ (SAHMRI), North Terrace, Adelaide, South Australia, Australia. ${ }^{3}$ Ironwood Pharmaceuticals, Cambridge, Massachusetts, \\ USA. ${ }^{4}$ Interfakultäres Institut für Biochemie, University of Tübingen, Tübingen, Germany. ${ }^{5}$ AnaBios, San Diego, California,

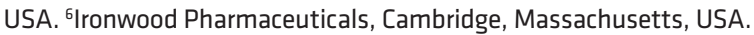

Irritable bowel syndrome (IBS) patients suffer from chronic abdominal pain and extraintestinal comorbidities, including overactive bladder (OAB) and interstitial cystitis/painful bladder syndrome (IC-PBS). Mechanistic understanding of the cause and time course of these comorbid symptoms is lacking, as are clinical treatments. Here, we report that colitis triggers hypersensitivity of colonic afferents, neuroplasticity of spinal cord circuits, and chronic abdominal pain, which persists after inflammation. Subsequently, and in the absence of bladder pathology, colonic hypersensitivity induces persistent hypersensitivity of bladder afferent pathways, resulting in bladder-voiding dysfunction, indicative of OAB/IC-PBS. Daily administration of linaclotide, a guanylate cyclase-C (CC-C) agonist that is restricted to and acts within the gastrointestinal tract, reverses colonic afferent hypersensitivity, reverses neuroplasticity-induced alterations in spinal circuitry, and alleviates chronic abdominal pain in mice. Intriguingly, daily linaclotide administration also reverses persistent bladder afferent hypersensitivity to mechanical and chemical stimuli and restores normal bladder voiding. Linaclotide itself does not inhibit bladder afferents, rather normalization of bladder function by daily linaclotide treatment occurs via indirect inhibition of bladder afferents via reduced nociceptive signaling from the colon. These data support the concepts that crossorgan sensitization underlies the development and maintenance of visceral comorbidities, while pharmaceutical treatments that inhibit colonic afferents may also improve urological symptoms through common sensory pathways.

Conflict of interest: PG, CH, CBK, and ISS are, or were, employees of Ironwood Pharmaceuticals and own stock/stock options in Ironwood Pharmaceuticals. SMB received grant support from Ironwood Pharmaceuticals to conduct the study.

Submitted: April 24, 2018 Accepted: August 29, 2018 Published: October 4, 2018

Reference information: JCI Insight. 2018;3(19):e121841 https://doi.org/10.1172/jici. insight.121841.

\section{Introduction}

Irritable bowel syndrome (IBS) is a prevalent functional gastrointestinal disorder affecting approximately $11 \%$ of the global population (1). IBS places a significant financial burden on society and negatively affects the quality of life of those affected $(1,2)$. IBS is characterized by chronic abdominal pain or discomfort associated with altered bowel habits and is subclassified as IBS with constipation (IBS-C), IBS with diarrhea (IBS-D), and alternating/ mixed IBS (A/M-IBS) $(1,2)$. Abdominal pain is a key clinical feature of IBS and, paradoxically, is the most difficult symptom to treat $(1,2)$. Opioids are frequently used for pain management, yet their chronic use causes tolerance, dependence, and reduced analgesic efficacy, resulting in the current "opioid epidemic" (3). Importantly, chronic opioid use causes severe constipation, making opioid use in patients who already have constipation, such as the $33 \%$ of IBS patients who have IBS-C, problematic. Accordingly, opioids are not efficacious in treating visceral pain, and new analgesic treatments for visceral pain syndromes such as IBS are urgently required.

We have shown that linaclotide, an FDA-approved, synthetic, 14-amino acid peptide agonist of guanylate cyclase-C (GC-C), improves abdominal pain and bowel symptoms in IBS-C patients $(4,5)$. Linaclotide is minimally absorbed and acts locally within the gastrointestinal tract on GC-C expressed on the 
luminal side of intestinal epithelial cells $(4,6,7)$. In preclinical models, this interaction elevates intracellular and extracellular levels of cyclic GMP (cGMP), inducing fluid secretion and accelerating intestinal transit $(4,6,8)$. Preclinical models also show that a single dose of linaclotide can induce acute analgesia $(4,9)$. This effect is proposed to be mediated by the downstream effector of GC-C, cGMP, released basolaterally from colonic epithelial cells via cGMP efflux pumps, which inhibits colonic nociceptors by a mechanism that is currently unclear but being actively investigated (4).

Notably, the number of linaclotide-treated IBS-C patients reporting a reduction in abdominal pain actually increases over time (4). In phase III clinical trials, approximately $30 \%$ of IBS-C patients had a $>30 \%$ reduction in abdominal pain compared with baseline after 1 week of linaclotide treatment. This reduction in abdominal pain increased to $>50 \%$ of linaclotide-treated patients by week 3 and $>60 \%$ of linaclotide-treated patients by week 7 and was sustained at approximately $70 \%$ of linaclotide-treated patients for the remainder of the 26 weeks of treatment (4). However, the mechanisms underlying the enhanced analgesic effects following long-term linaclotide treatment remain unclear. Although the pathophysiology of IBS is not completely understood, hallmarks of IBS include allodynia and hyperalgesia to mechanical events within the intestine in the absence of overt pathology to the intestinal mucosa, suggesting neuroplasticity of colonic afferent pathways in the development and maintenance of chronic abdominal pain in IBS $(1,2,10)$. Accordingly, we hypothesized that chronic linaclotide treatment and its downstream effector, intestinal epithelial cell-derived cGMP, may be responsible for persistent inhibition of colonic nociceptors, a reduction in peripheral afferent drive to the spinal cord, and, ultimately, reversal of the neuroplasticity underlying chronic abdominal pain.

In addition to chronic abdominal pain, IBS patients also suffer from extraintestinal comorbidities, including overactive bladder (OAB) and interstitial cystitis/painful bladder syndrome (IC-PBS) $(1,11,12)$. Of particular interest is the observation that bladder dysfunction is significantly more common among IBS patients than in healthy control subjects $(1,13-17)$. Correspondingly, IBS patients are more likely to report $\mathrm{OAB}$ symptoms, including nocturia, urgency, and urge incontinence $(11,13)$. These reports are intriguing, as the colon and bladder are both innervated by spinal afferents, which have peripheral endings within their host tissue, axons that travel via the splanchnic and pelvic nerves, cell bodies located within the thoracolumbar (TL) and lumbosacral (LS) dorsal root ganglia (DRG), and central projections that synapse into the dorsal horn of the TL and LS spinal cord (11). Subconscious coordination of sensory signals from the colon and bladder via these spinal pathways is essential for synchronized defecatory and micturition responses (11). Therefore, disruption of these intimate neural processes could result in comorbid conditions, such as IBS and OAB/IC-PBS (11). Animal models have shown that active colitis causes transient bladder overactivity and bladder afferent dysfunction $(11,14-17)$. However, the long-term effects of colitis on bladder function have not been correlated to chronic neuroplasticity of bladder afferent pathways. Furthermore, once established, it remains unclear if bladder dysfunction can be normalized with the use of therapeutic treatments. We hypothesized that colitis-induced neuroplasticity of colonic afferent pathways subsequently causes chronic hypersensitivity of afferent pathways innervating the bladder. We also hypothesized that chronic linaclotide administration to reduce peripheral sensory drive from the colon may therefore subsequently reverse bladder afferent hypersensitivity and restore normal bladder function.

Here, we demonstrate that colitis induces neuroplasticity in sensory pathways innervating the colon and then subsequently the bladder, resulting in concurrent chronic abdominal pain and bladder dysfunction in postinflammatory states in mice. Chronic linaclotide treatment reverses this neuroplasticity via a cGMP-dependent extracellular mechanism, alleviating abdominal pain and normalizing bladder function. These findings suggest that improving abdominal pain with linaclotide treatment, which is localized to and acts via GC-C within the gastrointestinal tract, may improve urological symptoms through inhibition of sensory pathways common to the colon and bladder.

\section{Results}

Colitis induces chronic allodynia and hyperalgesia, which is reversed by chronic daily linaclotide treatment. In order to determine if chronic linaclotide treatment ( $3 \mu \mathrm{g} / \mathrm{kg}$ by $100 \mu \mathrm{l}$ oral gavage, ref. 9 , once daily for 2 weeks) reduces chronic abdominal pain, we used an IBS mouse model of chronic visceral hypersensitivity (CVH). These mice were administered intracolonic trinitrobenzenesulphonic acid (TNBS) and developed colitis, as indicated by macroscopic damage, crypt segmentation, and edema to the colonic epithelium, in addition to significant increases in myeloperoxidase (MPO) activity (Supplemental Figure 1; supplemental material 
available online with this article; https://doi.org/10.1172/jci.insight.121841DS1). While colonic inflammation spontaneously healed over a 7-day period (Supplemental Figure 1), these mice subsequently developed chronic colonic afferent mechanical hypersensitivity in the postinflammatory state $(4,18-21)$. First, we showed that $\mathrm{CVH}$ mice administered vehicle (saline oral gavage, once daily for 2 weeks, from 14 to 28 days after intracolonic TNBS administration) displayed allodynia and hyperalgesia in response to colorectal distension (CRD) relative to control mice administered vehicle (Figure 1A). This indicates that the process of chronic oral gavaging mice per se does not interfere with the development of CVH. Next, we showed that control mice administered either chronic daily linaclotide or vehicle displayed similar pain responses to $\mathrm{CRD}$ across a range of pressures (Figure 1B). In contrast, $\mathrm{CVH}$ mice chronically administered daily linaclotide showed significantly reduced pain responses to CRD compared with CVH mice administered vehicle (Figure 1C). These changes were not associated with alterations in colonic compliance (Supplemental Figure 2), suggesting that they are mediated at the level of the primary afferent, rather than secondary to changes in the ability of the colon to accommodate an increase in volume. Overall, CVH mice administered daily linaclotide exhibited similar pain responses to those of control animals (Figure 1, B-D), suggesting that chronic linaclotide treatment reverses the allodynia and hyperalgesia normally apparent in CVH states.

Colitis induces chronic hypersensitivity of colonic nociceptors, which is reversed by chronic daily linaclotide treatment. To determine if the linaclotide-induced reversal of allodynia and hyperalgesia in CVH states is due to long-term inhibition of peripheral sensory drive from the colon we performed ex vivo recordings of colonic nociceptors from mice administered either chronic linaclotide or vehicle. Colonic nociceptors from $\mathrm{CVH}$ mice displayed increased responsiveness to mechanical stimuli $(4,18-21)$. We found that control mice administered daily linaclotide displayed modest reductions in colonic afferent responses to mechanical stimuli relative to those of vehicle-treated control mice (Figure 1, E and F). In contrast, colonic nociceptors from $\mathrm{CVH}$ mice administered daily linaclotide for 2 weeks showed pronounced decreases in mechanical responsiveness (Figure 1, G and $\mathrm{H}$ ) and normalized activation thresholds (Supplemental Figure 3) relative to those of vehicle-treated $\mathrm{CVH}$ mice. These findings suggest that chronic linaclotide treatment reverses the chronic mechanical hypersensitivity of colonic nociceptors normally observed in CVH states.

Colitis induces neuroplasticity within the dorsal horn of the spinal cord, which is reversed by chronic daily linaclotide treatment. We next assessed if the linaclotide-induced suppression in peripheral drive from the colon resulted in corresponding changes in nociceptive signaling within the dorsal horn of the spinal cord. To do this, we identified activated dorsal horn neurons in response to noxious ( $80 \mathrm{mmHg}) \mathrm{CRD}$ by phosphorylated MAP kinase ERK 1/2 immunoreactivity (pERK-IR). CVH mice treated with vehicle displayed significantly more activated dorsal horn neurons in response to noxious CRD compared with vehicle-treated $\mathrm{CVH}$ mice (Figure $2 \mathrm{~A}$ ). In control mice, daily linaclotide treatment did not alter the number of activated dorsal horn neurons in response to noxious CRD relative to that in vehicle-treated control mice (Figure 2, A and B). In contrast, daily linaclotide treatment in CVH mice resulted in significantly fewer pERK-IR dorsal horn neurons after noxious CRD compared with vehicle-treated $\mathrm{CVH}$ mice (Figure 2, A and C). This decrease was particularly apparent within lamina I, II, and V of the dorsal horn, with the number of activated neurons similar to that in control conditions (Figure 2). In order to determine if different populations of pERK-IR neurons were activated in CVH states, we colabeled for calbindin (excitatory neurons, ref. 22) or GABA (inhibitory interneurons, ref. 22; Supplemental Figure 4). While the number of $\mathrm{pERK}$-IR neurons expressing calbindin did not change between conditions and treatments (Supplemental Figure 4, A and B), we observed a significant increase in the percentage of pERK-IR dorsal horn neurons expressing GABA (Supplemental Figure 4, C and D). This effect was partially reversed in $\mathrm{CVH}$ mice treated with linaclotide.

We have previously shown that $\mathrm{CVH}$ mice display sprouting within the spinal cord of the central terminals of colon-innervating afferents, particularly within lamina I but also deeper laminae (23). We therefore sought to determine if the linaclotide-induced decrease in peripheral sensory drive from the colon, plus the reduced activation of dorsal horn neurons in response to noxious $\mathrm{CRD}$, would alter sprouting of colonic afferent central terminals in $\mathrm{CVH}$ states. We found that, relative to control animals, $\mathrm{CVH}$ vehicle-treated mice displayed an increased optical density (Figure 3A) and area covered by colonic afferent central terminals in the dorsal horn (laminae I and II) (Figure 3B), as identified by fluorescent retrograde labeling of colon-innervating neurons using CTB-555 (Figure 3C and Supplemental Figure 5). CVH mice treated daily with linaclotide showed a reduced density and distribution of colonic afferent central terminals, suggesting sprouting of these terminals within the spinal cord was not as extensive as that normally observed in $\mathrm{CVH}$ 
A
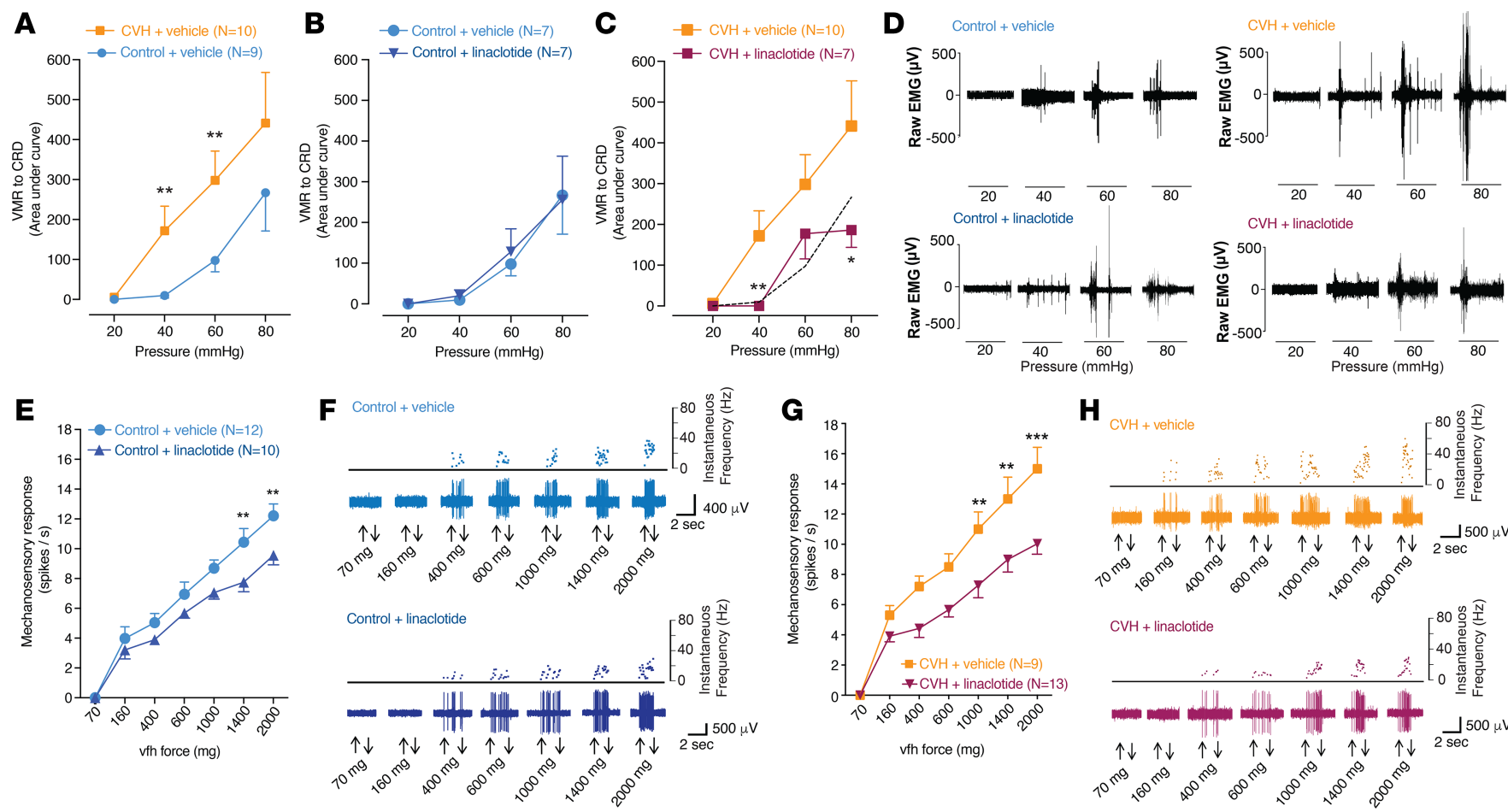

$\mathbf{H}_{\mathrm{OHH}+\text { venide }}$

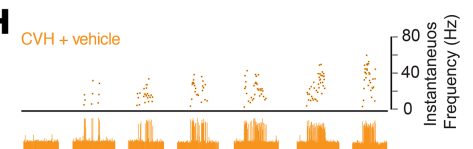$$
\uparrow \downarrow \uparrow \downarrow \uparrow \downarrow \uparrow \downarrow \uparrow \downarrow \uparrow \uparrow \downarrow \uparrow \downarrow \frac{2 \sec }{4} 500 \mu
$$

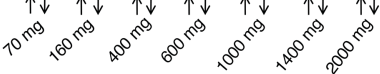

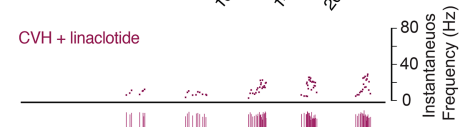

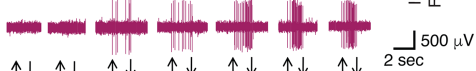

$\uparrow \downarrow \uparrow \downarrow \uparrow \downarrow \quad \uparrow \downarrow \uparrow \uparrow \downarrow \uparrow \downarrow \quad \uparrow \downarrow 2 \frac{\sec }{500}$

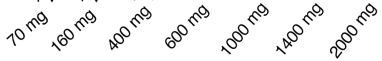

Figure 1. Chronic oral administration of linaclotide reduces colonic nociception and reverses chronic visceral hypersensitivity. (A) Chronic visceral hypersensitivity $(\mathrm{CVH})$ mice have enhanced visceromotor responses (VMRs) to colorectal distension (CRD) compared with those of control mice (** $P<$ 0.01). (B) Control mice orally administered linaclotide ( $3 \mu \mathrm{g} / \mathrm{kg}$ once daily for 14 days) show no difference in their VMR to CRD relative to vehicle-treated control mice. (C) CVH mice treated with chronic linaclotide (starting 14 days after intracolonic TNBS) display reduced VMRs relative to vehicle-treated CVH mice $\left({ }^{*} P<0.05,{ }^{*} P<0.01\right)$. The dashed line indicates the mean responses from controls. (D) Electromyographic (EMG) recordings of VMRs (20-80 mmHg, 20 -second duration, 4-minute intervals) in control and CVH mice orally administered vehicle or linaclotide ( $3 \mu \mathrm{g} / \mathrm{kg}$ ) once daily for 14 days. (E) Ex vivo single-fiber recordings of colonic nociceptors from linaclotide-treated control mice show reduced firing in response to higher forces of mechanical stimuli compared with vehicle-treated control mice $\left.{ }^{* *} P<0.01\right)$. (F) Ex vivo colonic nociceptor recording. Arrows indicate application/removal of von Frey hairs (vfh). (C) Colonic nociceptors from linaclotide-treated CVH mice display reduced firing in response to mechanical stimuli compared with vehicle-treated CVH mice ( $\left.{ }^{* *} P<0.01,{ }^{* * *} P<0.001\right)$. (H) Ex vivo nociceptor recordings from CVH mice treated with either vehicle or linaclotide. Arrows indicate application/ removal of von Frey hairs. Data represent mean \pm SEM. $P$ values were determined by generalized estimating equations, followed by least significant difference post hoc $(\mathbf{A}-\mathbf{C})$ or by 2-way ANOVA with post hoc Tukey's test (E and $\mathbf{G})$.

mice (Figure 3 and Supplemental Figure 5). Collectively, these findings suggest that daily linaclotide treatment reverses $\mathrm{CVH}$-associated neuroplasticity in colonic afferent pathways.

Exogenous cGMP inhibits colon-innervating DRG neurons via a membrane receptor target that is accessed extracellularly. Previous studies suggest that the GC-C agonist linaclotide does not directly inhibit nociceptors, rather the downstream effector of GC-C, cGMP released basolaterally from colonic epithelial cells, inhibits colonic nociceptors (4). However, this mechanism and the manner in which cGMP exerts these inhibitory actions remain to be fully elucidated. Using whole-cell patch-clamp electrophysiology of colon-innervating DRG neurons, we confirmed that linaclotide does not affect the excitability of these neurons (Figure 4, A and B). Colon-innervating DRG neurons from CVH mice displayed pronounced neuronal hyperexcitability relative to that of control counterparts (Figure 4C). In contrast to linaclotide, exogenous application of cGMP inhibited a large population of colon-innervating DRG neurons, particularly in CVH states (Figure 4, D-F). In CVH DRG neurons, cGMP caused a dose-dependent inhibition of neuronal excitability, significantly increasing the amount of injected current required to fire an action potential (Figure 4, F and G). Importantly, cGMP was still able to inhibit colonic nociceptors from mice

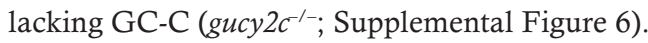

Having confirmed that cGMP, rather than linaclotide, inhibits colonic nociceptors, the question remained as to the underlying mechanism. Although active mechanisms for transport of cGMP out of cells have been described, passive diffusion of cGMP across cell membrane is poor, and cGMP is not actively transported back into cells (24). We therefore hypothesized that the effects of cGMP on colonic nociceptors are via a membrane target accessible extracellularly. To investigate this, we utilized fluorescence resonance energy transfer-based 
A

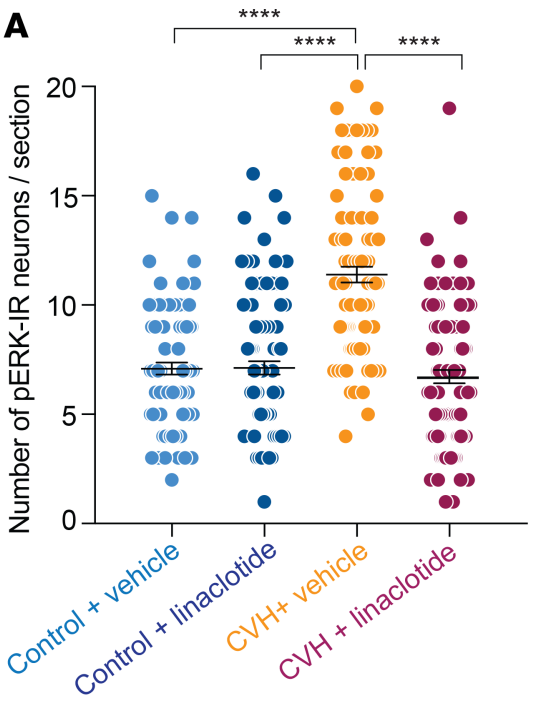

Figure 2. Chronic oral administration of linaclotide reduces the activation of dorsal horn neurons within the spinal cord. (A) Noxious ( $80 \mathrm{mmHg}$ ) colorectal distension (CRD) activates dorsal horn neurons within the thoracolumbar spinal cord, indicated by phosphorylated MAP kinase ERK $1 / 2$ immunoreactivity (pERK-IR). Dots indicate individual counts in spinal cord sections from $N=$ 6-11 mice per group. In response to noxious CRD, chronic visceral hypersensitivity $(\mathrm{CVH})$ mice $(N=10)$ display more activated neurons within the thoracolumbar spinal cord regions T10-L1 relative to control mice administered vehicle $\left({ }^{* * *} P<0.0001, N=11\right)$ or linaclotide $\left({ }^{* * *} P<0.0001, N=6\right)$. CVH mice chronically administered linaclotide $(N=7)$ displayed fewer activated neurons in response to noxious CRD within T10-L1 compared with vehicle-treated CVH mice $\left({ }^{* * *} P<0.0001\right)$. Histological sections of pERK-IR within the spinal cord of (B) control and (C) CVH mice chronically administered vehicle or linaclotide. Yellow arrows indicate $p E R K-I R$ neurons. Scale bars: $100 \mu \mathrm{m}$. Data represent mean \pm SEM. $P$ values are based on 1-way ANOVA with Dunn's multiple comparison tests.
B
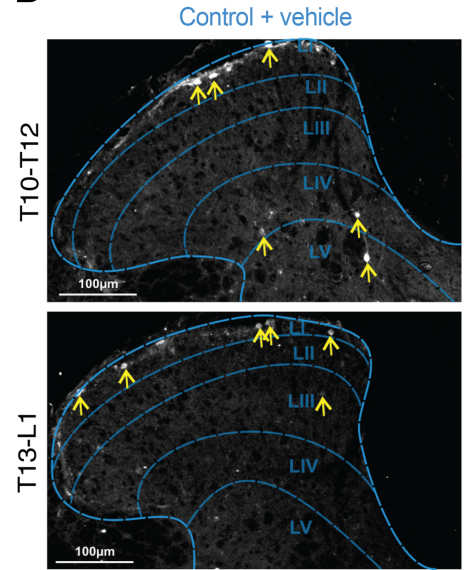
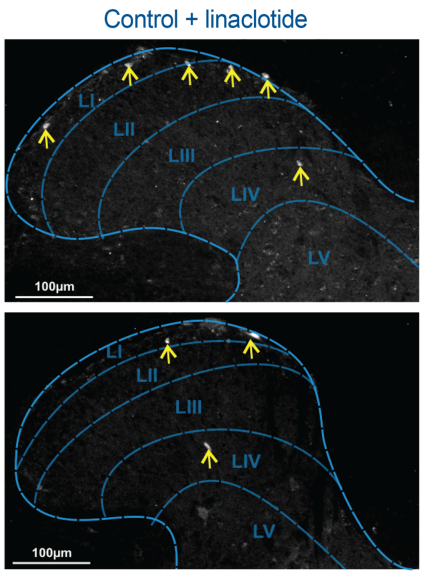

C
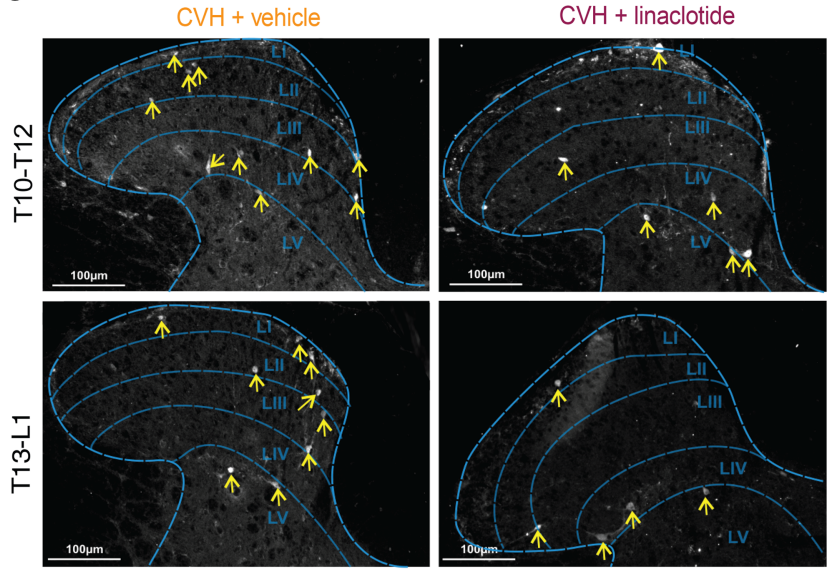

(FRET-based) analysis of genetically encoded fluorescent biosensors for cGMP. DRG neurons were isolated from R26-CAG-mcGi500(L1) mouse embryos, which express the membrane-targeted version of cGi500, a cGMP indicator with an $\mathrm{EC}_{50}$ of $500 \mathrm{nmol} / 1$ that displays fast binding kinetics and exquisite selectivity for cGMP (25-27). Application of C-type natriuretic peptide (CNP; used as a positive control, as it results in the intracellular production of cGMP) to R26-CAG-mcGi500 DRG neurons increased the CFP/YFP emission ratio in the cell soma (Figure 4, H, J, and L) and growth cones (Figure 4, I, K, and M), which is a measure of the intracellular cGMP concentration. In contrast, uroguanylin (an endogenous GC-C agonist) did not increase intracellular cGMP concentrations in either DRG soma (Figure 4, J and L) or growth cones (Figure 4, $\mathrm{K}$ and $\mathrm{M}$ ), confirming a lack of functional GC-C expression by DRG neurons. Adding increasing concentrations of exogenous extracellularly applied cGMP did not change FRET ratio signals in either DRG soma (Figure 4, J and L) or growth cones (Figure 4, K and M), suggesting that cGMP is unable to cross neuronal membranes. However, deliberate permeabilization of the membrane with $\beta$-escin treatment, followed by exogenous extracellular cGMP, caused increased FRET signal ratios in both DRG soma (Figure 4, J and L) and growth cones (Figure 4, $\mathrm{K}$ and $\mathrm{M}$ ), indicating that cGMP had accessed the intracellular space. Overall, these findings confirm that cGMP inhibits colonic nociceptors via a membrane target that is accessed extracellularly.

Human DRG neurons are also inhibited by exogenous cGMP. To further investigate the translatability of our findings, we tested the ability of exogenously applied cGMP to inhibit human DRG neurons from organ donors. Using whole-cell patch-clamp electrophysiology, we found that application of exogenous cGMP caused a dose-dependent decrease in the number of action potentials fired by human DRG neurons (Figure 5, A and B). In order to simulate a pathological state, we incubated a subset of the DRG neuronal cultures with an inflammatory soup (histamine: $10 \mathrm{M}$, PGE-II: $10 \mu \mathrm{M}$, serotonin: $10 \mu \mathrm{M}$, bradykinin: $10 \mu \mathrm{M}$ ) for 2 hours. 
A

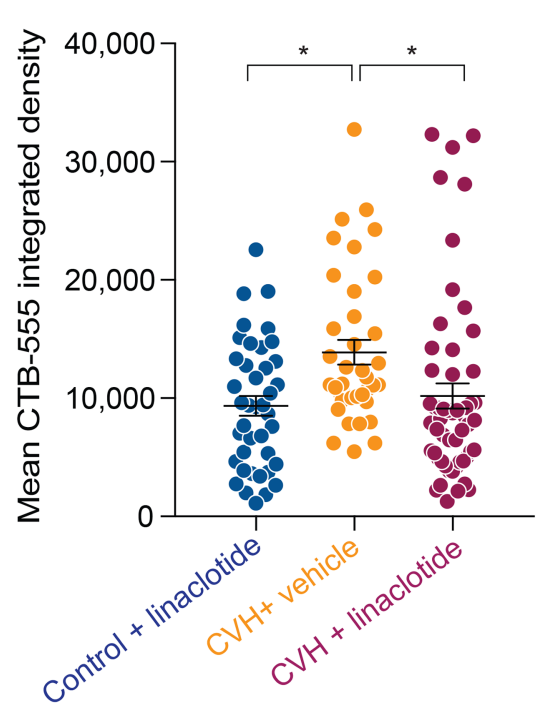

B

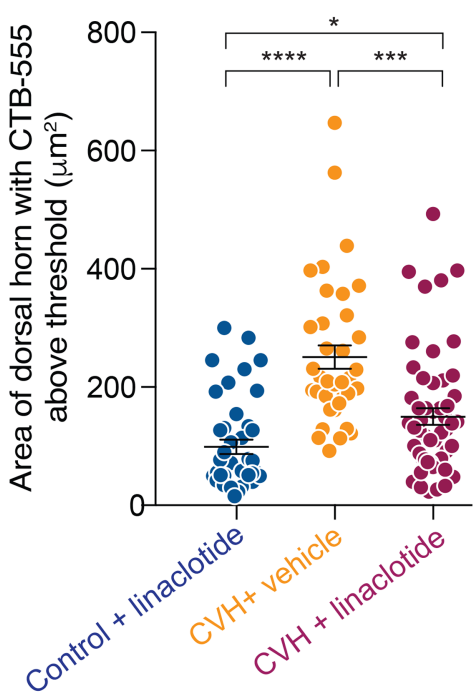

C
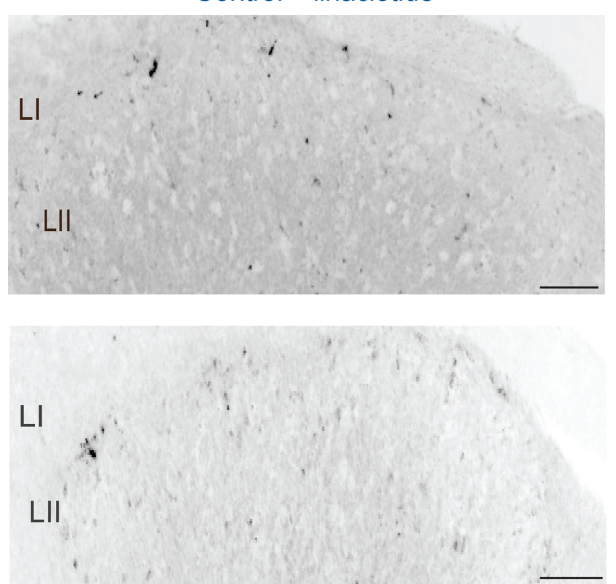

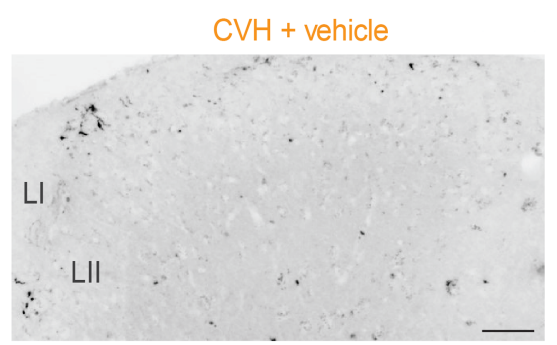

s.

LI

$T$.

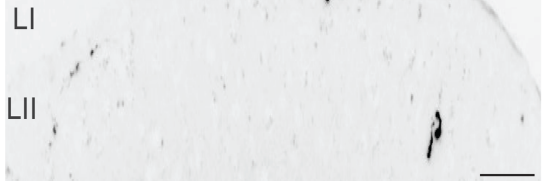

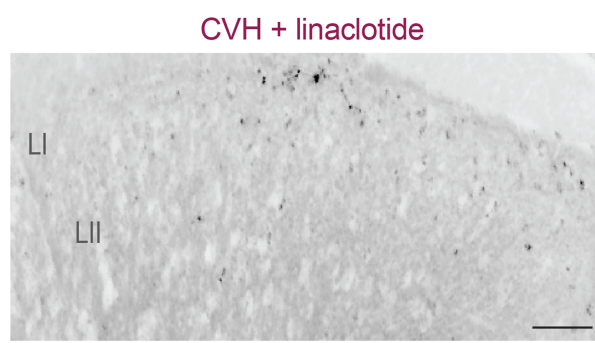

+ 4

$\mathrm{L}$

LII

Figure 3. Chronic oral administration of linaclotide reduces sprouting of colonic afferent terminals within the spinal cords of CVH mice. The central terminals of colon-innervating afferents within the thoracolumbar dorsal horn of the spinal cords of control and CVH mice were identified using retrograde tracing (CTB-555). Dots indicate individual spinal cord sections from $N=4$ mice per group. CVH mice display an increased intensity $\left(\mathbf{A}\right.$, ${ }^{*} P<$ $0.05)$ and density $\left(\mathbf{B},{ }^{* * *} P<0.0001\right)$ of retrograde tracer compared with that in control mice, suggesting sprouting of terminals during $\mathrm{CVH}$. Chronic linaclotide administration to CVH mice reduced tracer intensity $\left(\mathbf{A},{ }^{*} P<0.05\right)$ and density $\left(\mathbf{B},{ }^{* * *} P<0.001\right)$ compared with vehicle-treated $\mathrm{CVH}$ mice $(\mathrm{N}=$ 4 mice per group). (C) Sections of dorsal horn showing tracer accumulation within the central terminals of colon-innervating afferents from control mice chronically administered linaclotide or CVH mice administered vehicle or linaclotide. Images were converted to inverted monochromes, so that immunoreactivity appears black. Scale bars: $100 \mu \mathrm{m}$. Data represent mean \pm SEM. $P$ values are based on 1-way ANOVA with Dunn's multiple comparison tests.

Human DRG neurons from these cultures displayed pronounced hyperexcitability (Figure 5C), with application of exogenous cGMP causing a dose-dependent decrease in the number of action potentials fired (Figure 5, $\mathrm{D}$ and $\mathrm{E})$. Overall, more human DRG neurons from the inflammatory soup cultures were inhibited by cGMP compared with human DRG neurons from the normal cultures (Figure 5F).

Mice with colitis-induced CVH display altered bladder-voiding patterns, an effect that is reversed by chronic daily linaclotide treatment. We hypothesized that mice with CVH following spontaneous recovery from TNBS-induced colitis also exhibit altered bladder function, indicating the development of an OAB/IC-PBS phenotype. To investigate this, we examined mouse bladder-voiding patterns before and at 7, 14, 21, and 28 days following intracolonic TNBS treatment (Figure 6). Filter paper samples from the cages of control mice showed a distinctly organized pattern of voiding throughout the time course of the experiment, from day 1 through day 28 (Figure 6, A-E). In contrast, CVH mice began to develop a disrupted, scattered voiding pattern by day 14 after intracolonic TNBS, which was maintained and exacerbated by day 28 (Figure 6, B-J). 

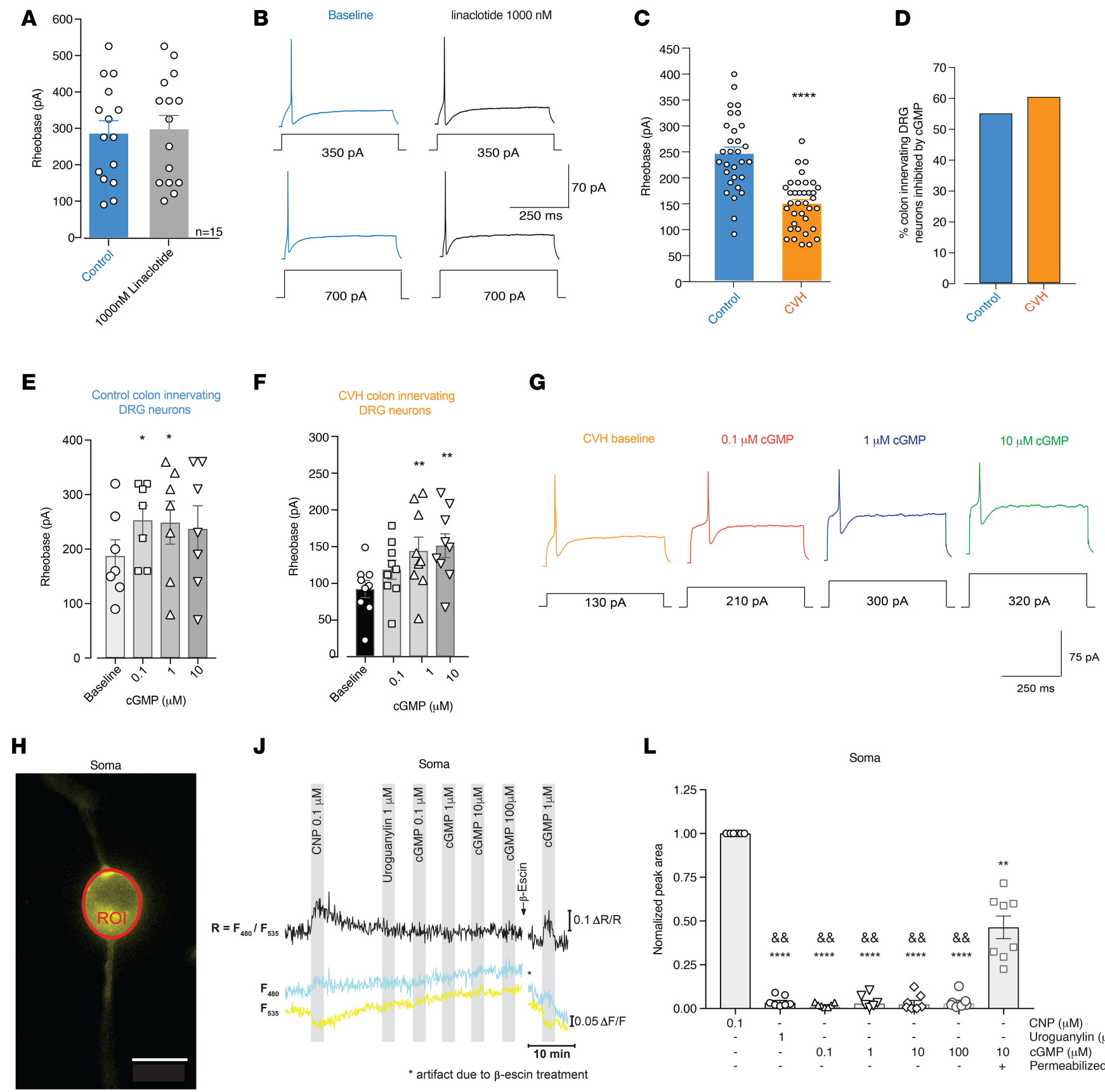

J
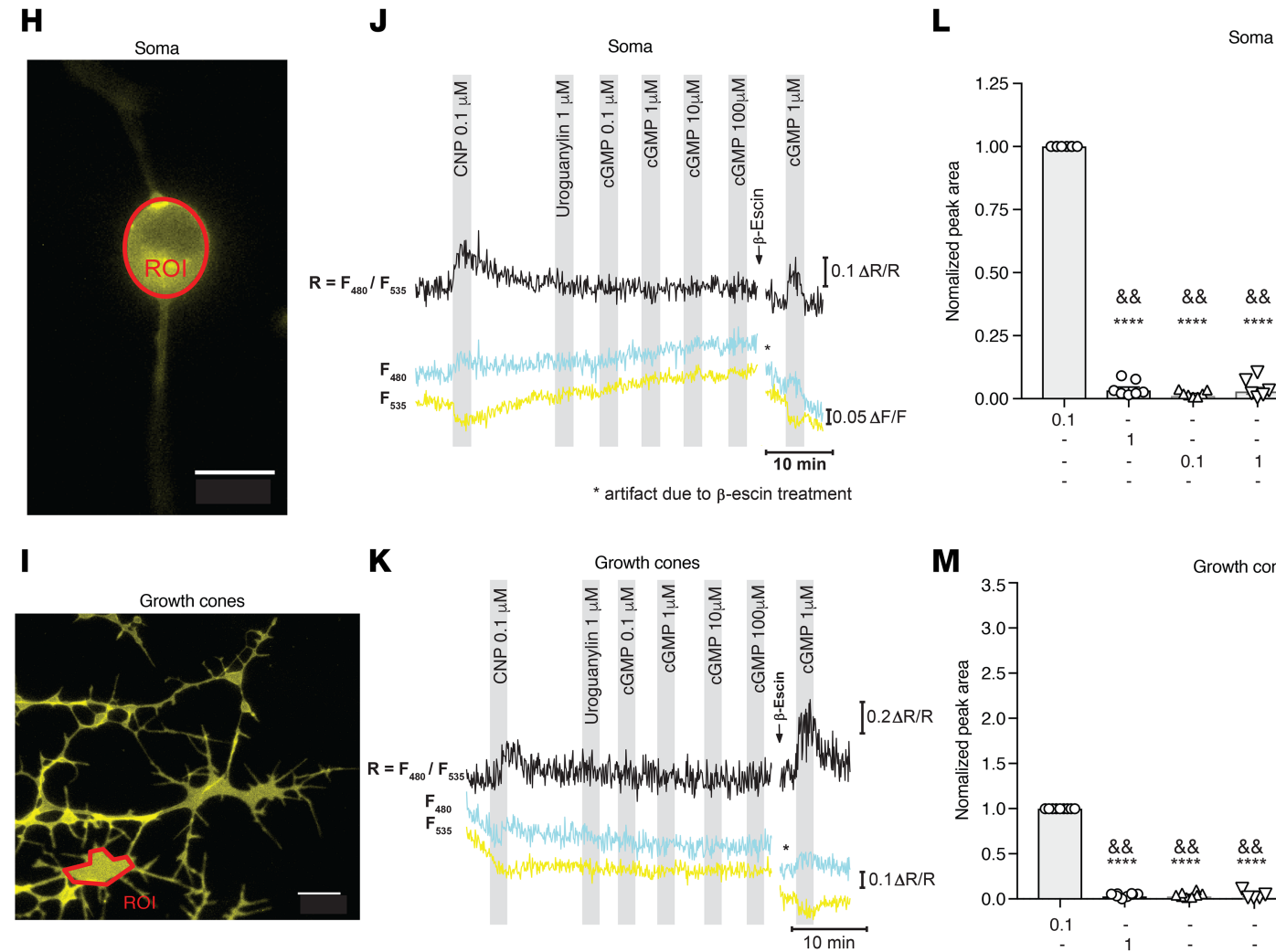

* artifact due to $\beta$-Escin treatment
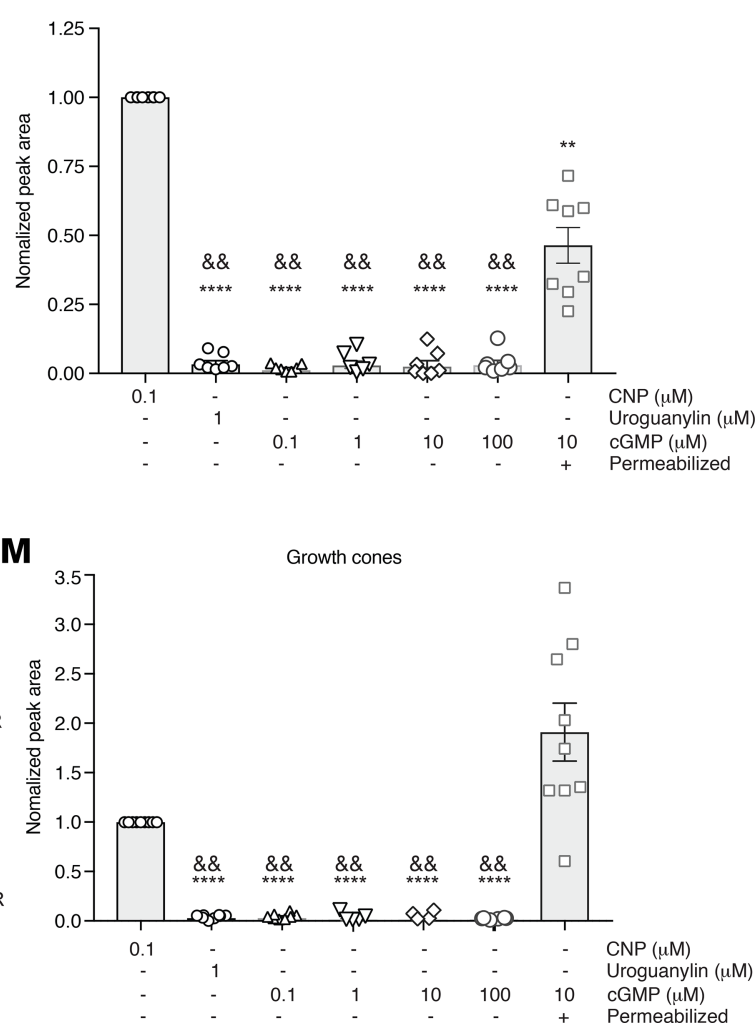
Figure 4. Exogenous cGMP inhibits colon-innervating DRG neurons via a membrane target accessed from an extracellular site. (A) Whole-cell current-clamp recordings of retrogradely traced colon-innervating DRG neurons $(n=15)$. Bath application of linaclotide $(1,000 \mathrm{nM})$ did not affect rheobase (amount of current required to fire an action potential). (B) Representative recordings in response to 500-millisecond current injection at rheobase (top) and $2 \times$ rheobase (bottom). Recordings were from the same neuron before and after linaclotide. (C) Colon-innervating DRG neurons from CVH mice $(n=36)$ display a reduced rheobase compared with controls $(n=30)$, indicating neuronal hyperexcitability $(* * * *<0.0001)$. (D) Percentage of colon-innervating DRG neurons from control and CVH mice inhibited by exogenous CGMP (0.1-10 $\mu \mathrm{M})$. (E) Exogenous CGMP increases the rheobase of colon-innervating DRG neurons from control mice $(n=7)$ at $0.1 \mu \mathrm{M} \mathrm{CGMP}\left({ }^{*} P<0.05\right)$ and $1 \mu \mathrm{M} \mathrm{CGMP}\left({ }^{*} P<0.05\right)$ and $(\mathbf{F})$ CVH mice $(n=9)$ at $1 \mu \mathrm{M}\left({ }^{* *} P<0.01\right)$ and $10 \mu \mathrm{M}$ cGMP $\left({ }^{* *} P\right.$ $<0.01)$. (C) Recordings from a CVH colon-innervating DRG neuron to 500-millisecond current injections, showing rheobase at baseline and in the presence of 0.1-10 $\mu$ M cGMP. (H) A dissociated E12.5 DRG neuron and (I) growth cones isolated from R26-CAG-mcGi500 mice, expressing the intracellular membrane-targeted mcGi500 (YFP fluorescence). In both examples, the red circle represents the region of interest (ROI) that was chosen for the measurement shown in J and K. Scale bars: $10 \mu \mathrm{m}$. Fluorescence resonance energy transfer (FRET)/CGMP measurements performed with embryonic (J) DRG neurons and (K) growth cones. An increase in the CFP/YFP emission ratio, $R=F_{480} / F_{535}$, indicates increased intracellular cGMP concentrations. FRET-based cGMP imaging was performed with drug stimulations (gray bars) in the following order: C-type natriuretic peptide (CNP; $0.1 \mu \mathrm{M})$, uroguanylin (UG; $1 \mu \mathrm{M})$, and $0.1,10$, and $100 \mu \mathrm{M}$ cGMP. CFP (cyan) and YFP (yellow) emissions were recorded and the ratio (black) built, which corresponds to the intracellular cGMP concentration. After deliberate cell permeabilization with $\beta$-escin, cells were perfused with $1 \mu \mathrm{M} \mathrm{cGMP}$ (positive control). Group quantification of cGMP signals, showing extracellular cGMP does not enter the (L) somata of intact dissociated DRG neurons nor (M) their growth cones (****P<0.0001; CNP vs. uroguanylin and CGMP $(0.1-100 \mu \mathrm{M}$. \&\&P $<0.01$; CGMP permeabilization vs. uroguanylin and CGMP $(0.1-100 \mu M)$. Data represent mean \pm SEM. $P$ values are based on paired $t$ tests (A), unpaired $t$ tests (C), or 1-way ANOVA with Tukey's multiple comparison tests (E, F, L,and $\mathbf{M})$.

At day 28, these changes were characterized by an increase in the number of small- and medium-sized urine spots (Figure 6, I and J) as well as an altered distribution throughout the cage, indicative of the OAB/ IC-PBS symptoms of urgency and frequency. In $\mathrm{CVH}$ mice due to receive either vehicle or linaclotide treatment, we saw a similar pattern of voiding dysfunction at day 14 (Figure 6, C, D, G, and H). However, following initiation of chronic oral linaclotide administration (started on day 14 after intracolonic TNBS), $\mathrm{CVH}$-induced changes in voiding patterns were attenuated, normalizing at day 28 both the distribution and the number of small- and medium-sized urine spots relative to vehicle-treated $\mathrm{CVH}$ mice (Figure 6, C-J). Notably, colitis did not induce local inflammation or changes in bladder histology at any time point tested following TNBS-induced colitis (Supplemental Figure 1), indicating that the changes we see in bladder-voiding patterns are not a direct result of local bladder inflammation.

CVH mice show enhanced bladder afferent responses to distension, which are reversed by chronic daily linaclotide treatment. To understand the mechanisms underlying the changes in bladder-voiding parameters observed in CVH mice, we next sought to examine bladder afferent responses to bladder distension. Multiunit ex vivo pelvic afferent recordings from control mice showed that, as pressure within the bladder increased, there was a corresponding increase in action potential discharge (Figure 7, A and B). However, bladder afferents from $\mathrm{CVH}$ mice displayed a significantly enhanced response to distension compared with control mice, particularly at $10-30 \mathrm{mmHg}$ of bladder distension, pressures that are likely to trigger bladder voiding in states of OAB/IC-PBS (Figure 7, A and B). This suggests that the aberrant bladder-voiding patterns we observed in the current study could be mediated by changes in bladder afferent mechanosensitivity. Furthermore, by examining the pressure-volume relationship in the bladder during distension, we identified that there was no difference in muscle compliance (Supplemental Figure 7A) or contractility (Supplemental Figure 8A) between control and $\mathrm{CVH}$ mice. This suggests that the significant changes in bladder afferent mechanosensitivity in $\mathrm{CVH}$ mice are mediated at the level of the primary afferent, rather than secondary to changes in the ability of the bladder to accommodate an increase in volume. As we demonstrated that daily linaclotide is able to normalize bladder-voiding patterns and reduce colonic afferent sensitivity, we hypothesized that linaclotide may also be able to attenuate colitis-induced bladder hypersensitivity. Here, we show that $\mathrm{CVH}$ mice receiving daily linaclotide treatment had significantly reduced bladder afferent sensitivity to distension $(0-30 \mathrm{mmHg}$ ) compared with vehicle-treated CVH mice (Figure 7, C and D). However, chronic linaclotide administration had no effect on bladder muscle compliance (Supplemental Figure 7B) or bladder muscle contractility (Supplemental Figure 8B), demonstrating that linaclotide's effect on reducing bladder afferent mechanosensitivity is independent of altered muscle compliance or contractility.

Daily linaclotide treatment prevents colitis-induced increases in bladder afferent chemosensitivity. To further investigate the effects of the $\mathrm{CVH}$ state on bladder afferent function, we examined the effect of purinergic $\left(\mathrm{P} 2 \mathrm{X}_{3}\right)$, muscarinic, and transient receptor potential vanilloid 1 (TRPV1) receptor agonists on bladder afferent firing in control and $\mathrm{CVH}$ mice. Application of the individual agonists $\alpha \beta \mathrm{Me}-\mathrm{ATP}$, carbachol, or capsaicin, respectively, caused significant bladder afferent activity from control mice (Figure 7, E-G). However, afferent responses to these agonists were all significantly enhanced in bladder preparations from $\mathrm{CVH}$ mice (Figure 7, E-G). In contrast, CVH mice treated daily with linaclotide showed significantly reduced bladder 
A

Normal baseline
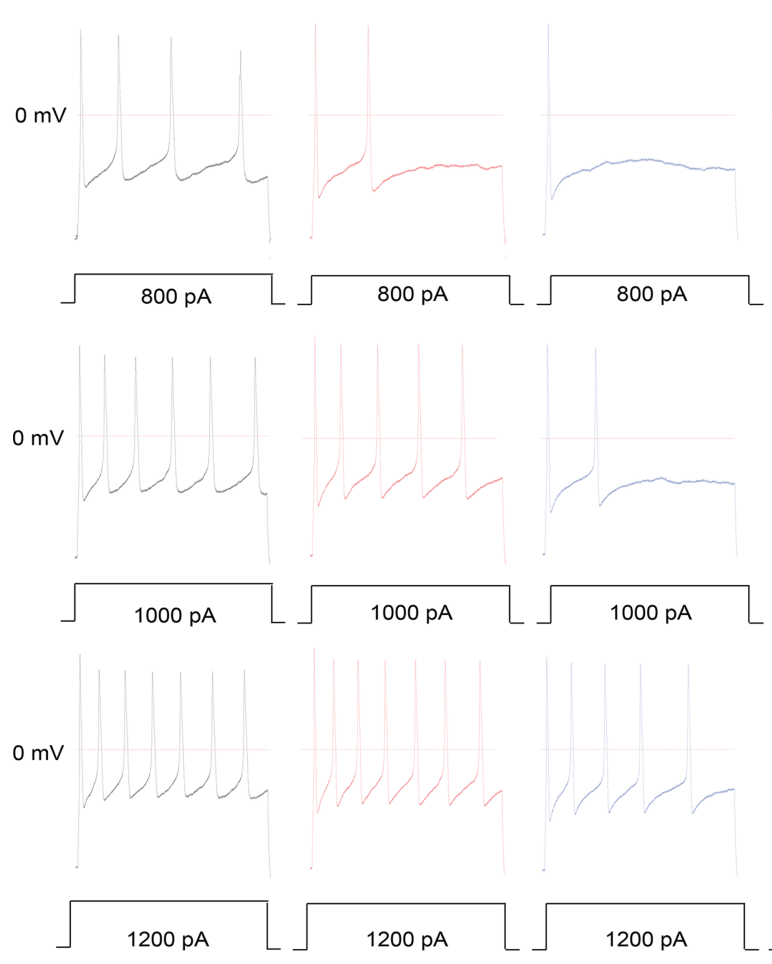

$1200 \mathrm{pA}$

\section{E}

Inflammatory soup

$0.1 \mu \mathrm{M}$ cGMP baseline
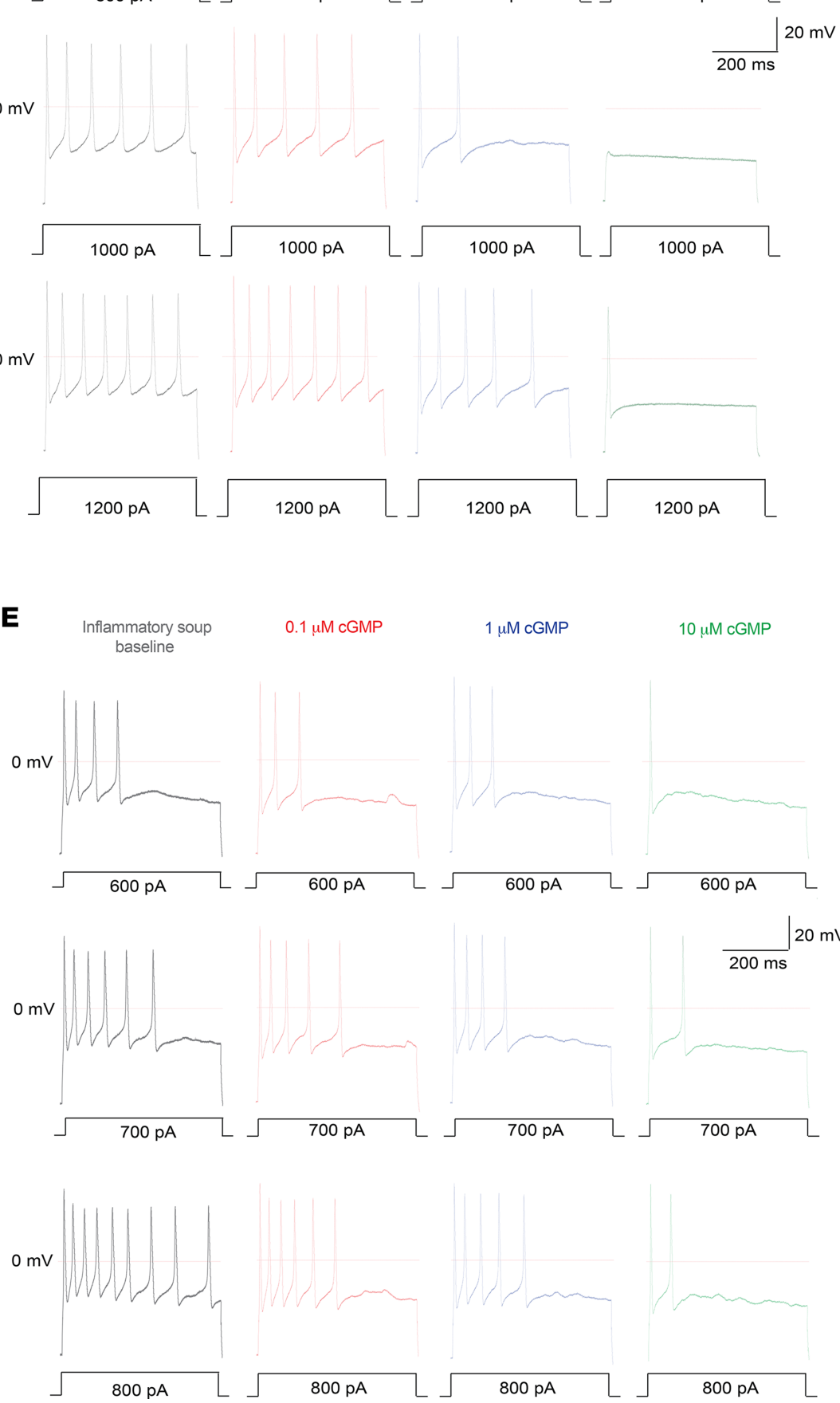

B
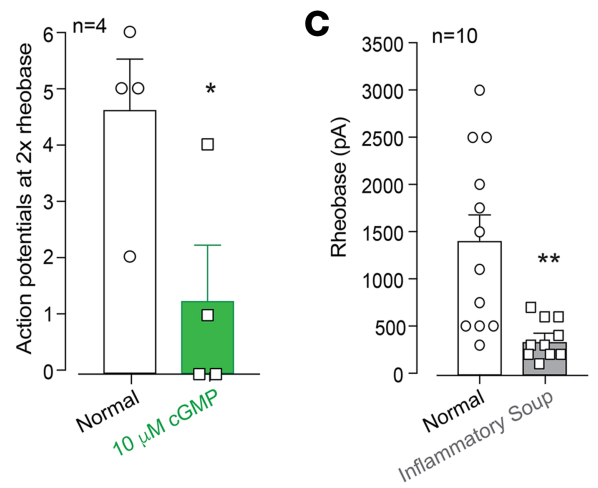

D

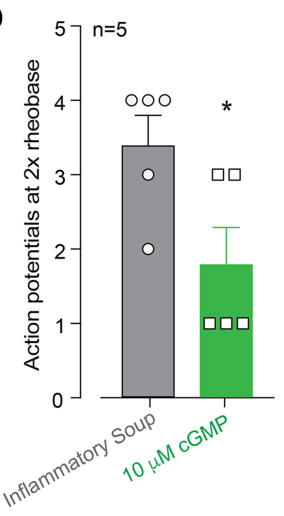

F

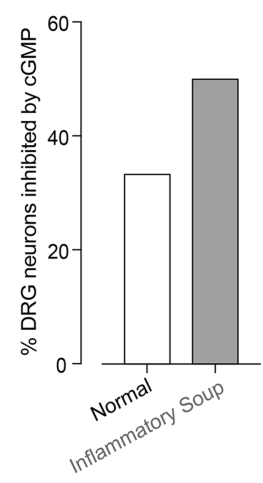

Figure 5. Exogenous cGMP inhibits human DRG neurons. (A) Whole-cell current-clamp recordings from a human DRG neuron, showing action potential firing in response to increasing current injections (800-1,200 pA, 500-ms duration). Increasing concentrations of exogenous cGMP $(0.1 \mu \mathrm{M}, 1 \mu \mathrm{M}$, and $10 \mu \mathrm{M}$ ) caused dose-dependent decreases in action potential firing. (B) Group data showing exogenous cGMP inhibits firing in affected neurons $\left({ }^{*} P<0.05\right)$.

(C) Human DRG neurons incubated for 2 hours prior to patching with an inflammatory soup displayed increased neuronal excitability, indicated by reduced rheobase $\left.{ }^{* *} P<0.01\right)$ compared with neurons in normal media. (D) Human DRG neurons incubated with inflammatory soup displayed reduced numbers of action potentials at $2 \times$ rheobase in the presence of cGMP (10 $\left.\mu \mathrm{M},{ }^{*} P<0.05\right)$. (E) Whole-cell current-clamp recordings from a human DRG neuron incubated in inflammatory soup at baseline and in the presence of exogenous cGMP (0.1-10 $\mu \mathrm{M})$. (F) More human DRG neurons incubated with inflammatory soup were inhibited by exogenous cGMP (10 $\mu \mathrm{M})$. Data represent mean \pm SEM. $P$ values are based on paired (B and $\mathbf{D}$ ) or unpaired $(\mathbf{C}) t$ tests.

afferent responses to $\alpha \beta \mathrm{Me}$-ATP, carbachol, and capsaicin compared with CVH vehicle-treated mice (Figure $7, \mathrm{H}-\mathrm{J})$, normalizing afferent responses to these agonists.

Chronic daily linaclotide reduces the excitability of bladder-innervating DRG neurons from CVH mice. In this study, we have shown that $\mathrm{CVH}$ mice display enhanced mechanical and chemical sensitivity of the peripheral sensory 
A
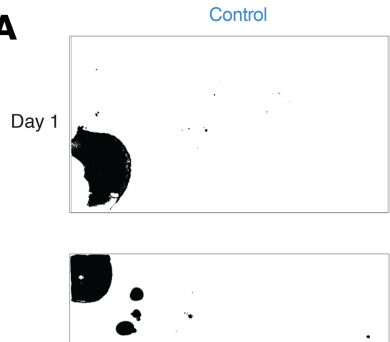

Day 7
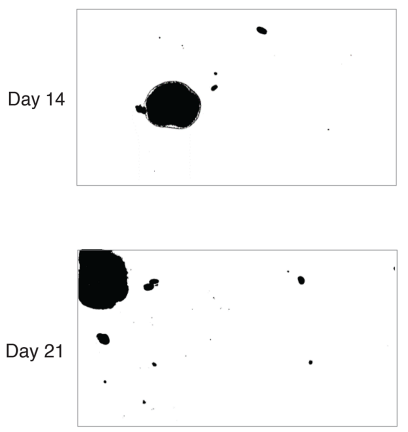

Day 28

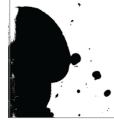

B

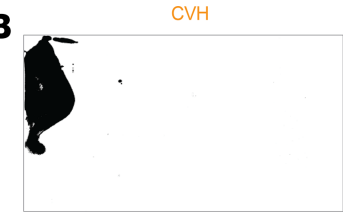

Intra-colonic TNBS administration
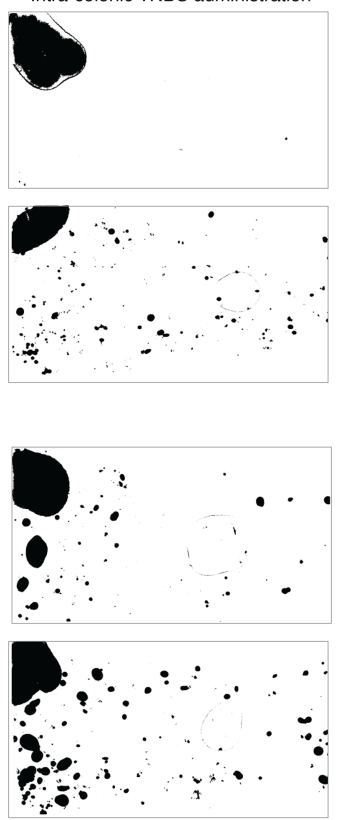

C

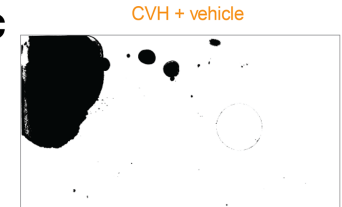

Intra-colonic TNBS administration
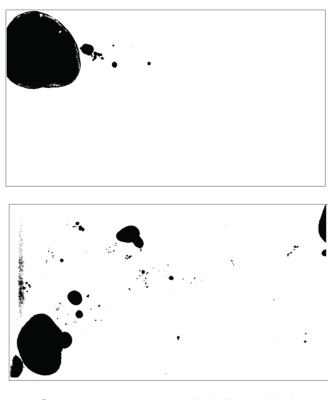

Commencement of daily vehicle treatment for 14 days

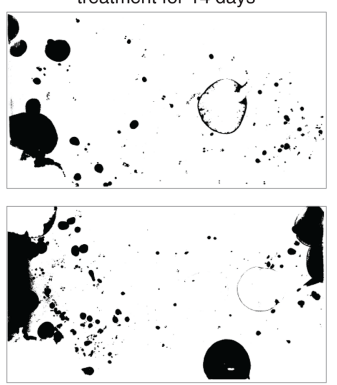

D

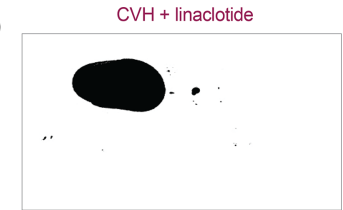

Intra-colonic TNBS administration
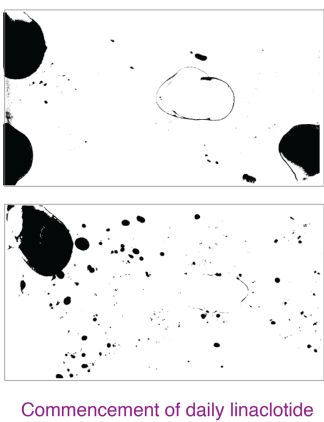

Commencement of daily linaclotide treatment for 14 day

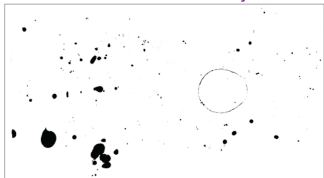

E

O Small Spots

Control

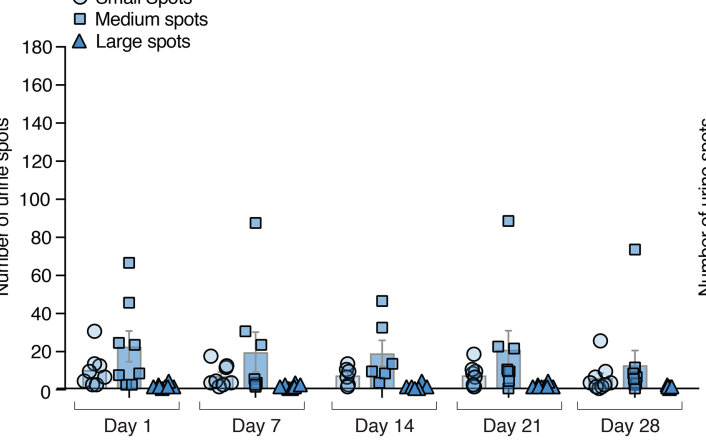

F Osmall Spots $\square$ Medium Spots

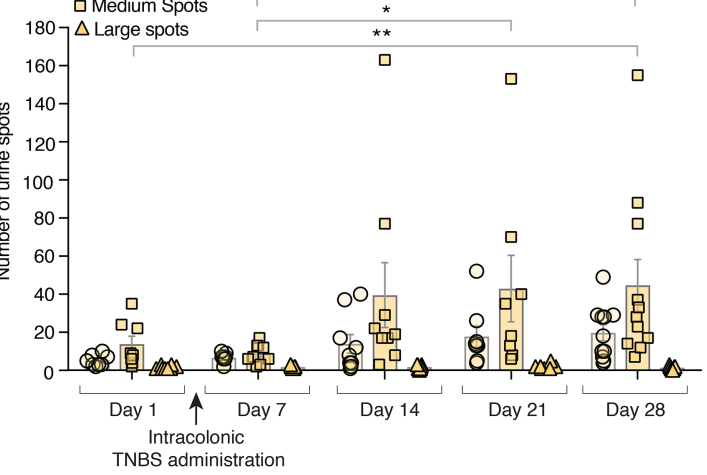

I

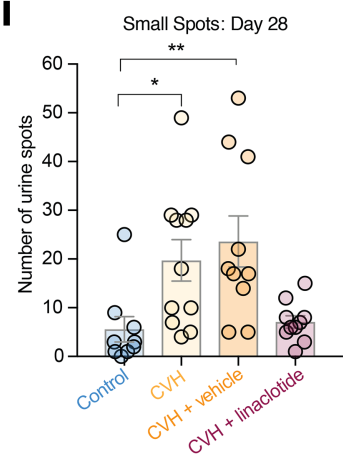

G

H
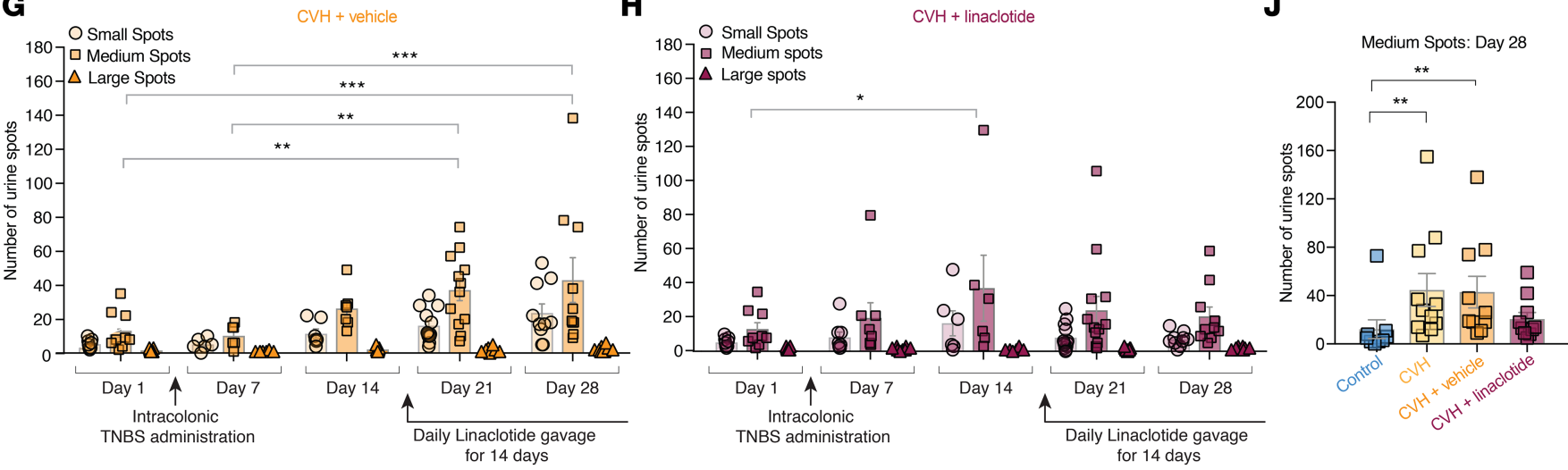

Figure 6. CVH mice display abnormal bladder voiding that is reversed by chronic oral administration of linaclotide. Filter paper images from individual mice followed for the duration of the 28-day study show $(\mathbf{A})$ control mice $(N=9)$ have predictable voiding patterns throughout, while $(\mathbf{B}) \mathrm{CVH}$ mice $(N=$ 11) and (C) CVH mice receiving vehicle treatment $(N=10)$ develop altered voiding patterns from day 14 after intracolonic TNBS. (D) By contrast CVH mice chronically administered linaclotide $(N=10)$ show normalized bladder-voiding patterns at 28 day. Scale bars: $10 \mathrm{~cm}$. (E) The number of small-, medium-, 
and large-sized urine spots produced by control mice is similar for each time point throughout the 28-day study. (F) CVH mice display increased numbers of medium-sized urine spots at 28 days after intracolonic TNBS administration compared with baseline (day 1; prior to TNBS, ${ }^{* *} P<0.01$ ) or 7 days after TNBS $\left({ }^{*} P<0.05,{ }^{* *} P<0.01\right)$. (G) CVH mice receiving vehicle treatment from $14-28$ days after TNBS display increased numbers of medium-sized spots at day $21\left({ }^{* *} P<0.01\right)$ and day 28 after intracolonic TNBS (*** $P$ 0.001). (H) CVH mice display more medium-sized urine spots at day 14 after TNBS ( ${ }^{*} P<0.05$ ), but, following chronic administration of linaclotide (from $14-28$ days after intracolonic TNBS), the number of medium-sized urine spots is normalized. (I and J) Quantitative data at day 28 for all treatment groups showing a significant increase in (I) small- and (J) medium-sized spots in CVH $\left({ }^{*} P<0.05,{ }^{* *} P<0.01\right)$ and $\mathrm{CVH}$ vehicle-treated $\left({ }^{*} P<0.01\right)$ mice compared with control mice. Data represent mean \pm SEM. $P$ values are based on 1 -way ANOVA followed by Bonferroni post hoc tests (E-J).

endings innervating the bladder and that these effects are independent of altered smooth muscle properties or local inflammation. To determine if this hypersensitivity occurs directly at the neuronal level, we used whole-cell patch-clamp electrophysiology to measure neuronal excitability of bladder-innervating DRG neurons. Retrogradely traced bladder-innervating TL and LS DRG neurons from CVH mice displayed pronounced hyperexcitability compared with those from control mice (Figure 8, A-C, and Supplemental Figure 9). Daily linaclotide treatment of $\mathrm{CVH}$ mice prevented $\mathrm{CVH}$-induced hyperexcitability of bladder-innervating DRG neurons relative to vehicle-treated $\mathrm{CVH}$ mice (Figure 8, A-C, and Supplemental Figure 9). Overall, these findings indicate that colitis-induced sensitization of colonic afferents is able to sensitize the cell bodies of bladder afferents and that chronic administration of linaclotide can prevent bladder afferent hypersensitivity in CVH states.

The effects of linaclotide are not mediated via direct actions within the bladder. Although linaclotide has a very low oral bioavailability and its receptor, GC-C, has been shown to be almost exclusively expressed in the mucosa of the gastrointestinal tract (4), we wanted to confirm that the effects seen in this study were not mediated by direct actions of linaclotide upon the bladder. Using in situ hybridization, we confirmed our previous findings and showed that GC-C is highly expressed in colonic epithelial cells (Figure 8D). In contrast to the colon, expression of GC-C was not evident within the bladder (Figure 8D). To further confirm our findings, we performed qPCR analysis of mRNA isolated from either primary colonic epithelial cells or bladder urothelial cells. These studies revealed that the colonic mucosa contained abundant GC-C mRNA expression, whereas GC-C mRNA expression was absent from the bladder mucosa (Figure 8E). In contrast, TRPV4, known to be expressed in bladder urothelial cells (28), was abundantly expressed in bladder urothelium (Figure $8 \mathrm{E}$ ). To functionally confirm a lack of action of linaclotide within the bladder, we used ex vivo bladder afferent recordings and infused linaclotide intravesically into the bladder during ramp distension (Figure 8, F and G). Intravesical linaclotide had no effect on bladder mechanosensitivity to bladder distension in either control or CVH mice (Figure 8, $\mathrm{F}$ and $\mathrm{G})$. These findings support our hypothesis that the inhibitory effects of linaclotide treatment are not via a direct action within the bladder.

Crosstalk of colon and bladder afferents within the DRG and spinal cord. To investigate how bladder afferents become hypersensitive in CVH states and why CVH mice display bladder dysfunction, we performed concurrent retrograde tracing from the colon and bladder. This allowed us to determine if these organs are innervated by a substantial population of DRG neurons that have dichotomizing axons that project to both the colon and the bladder. We found that the vast majority ( $85 \%)$ of retrogradely traced DRG neurons in both control and CVH states contain only the tracer injected into the colon or the tracer injected into the bladder, with a small population of dual-labeled (15\%) DRG neurons identified (Figure 9, A-F). A direct comparison of the number of traced colon-only, bladder-only, or dual-traced neurons per ganglia showed no significant alterations in the populations between control and CVH states in either TL or LS DRG (Figure 9, C and D). Furthermore, the percentage of colon-innervating neurons that also innervated the bladder (dual traced) did not alter between healthy and $\mathrm{CVH}$ mice (Figure 9, E and F). However, within the DRG it is evident that individual bladder- or colon- or dual-innervating neurons are located within close proximity to one another (Figure 9, A and B), allowing potential "crosstalk" between these organs. In separate tracing studies, we investigated the central terminals of colon-innervating and bladder-innervating afferents by visualizing retrograde tracing from each organ. These data show that the colon-only and bladder-only central terminals lie in very close proximity to one another within the dorsal horn of the spinal cord, in particular within laminae I, the lateral spinal nucleus, and the lumbar dorsal column (Figure 9, G-J). This close proximity provides potential opportunities for these central terminals to communicate with one another, allowing crosstalk between these organs onto shared spinal cord circuits. These findings may well explain why IBS patients have comorbidities, including abdominal pain and OAB/IC-PBS. 
A

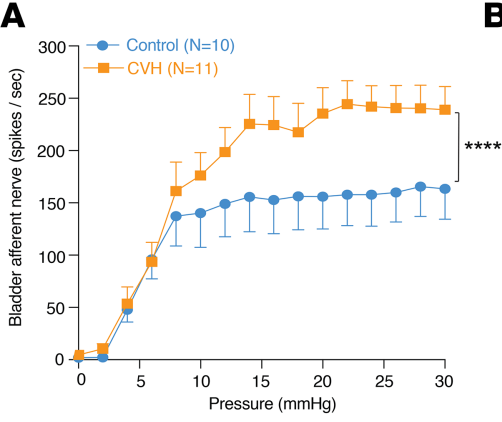

E

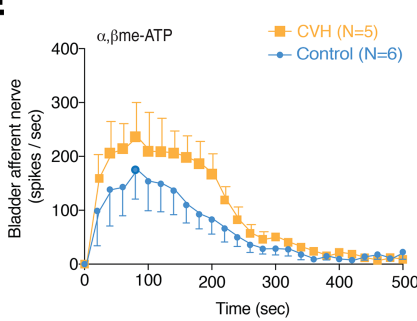

F

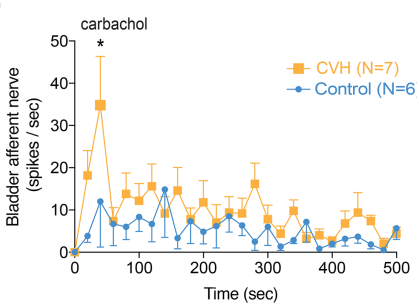

$\mathbf{G}$

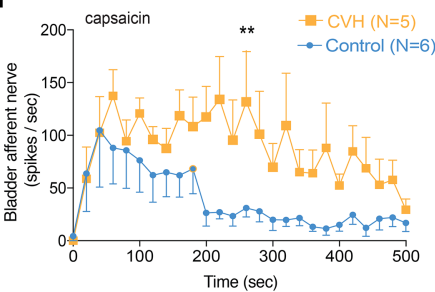

B

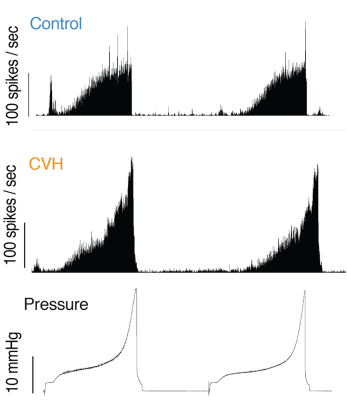

\& $\alpha, \beta$ me-ATP
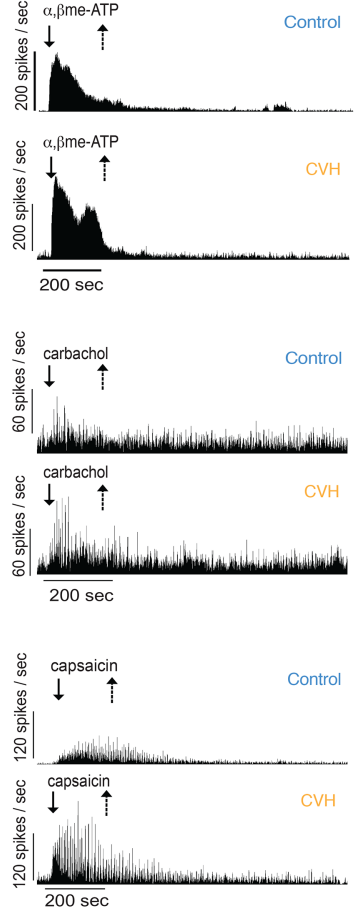

C

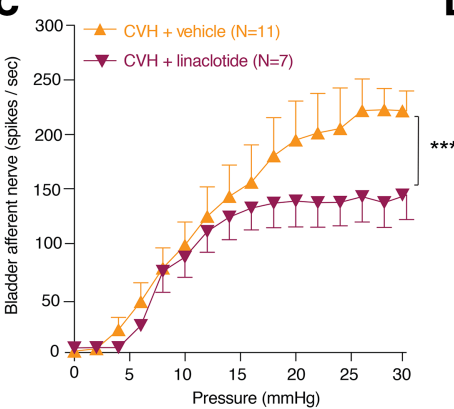

D

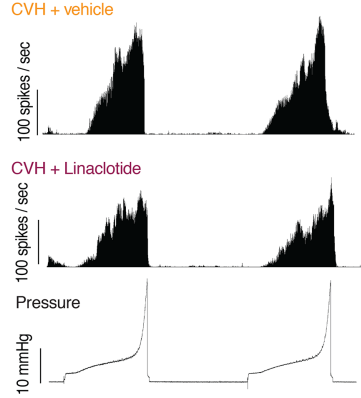

H
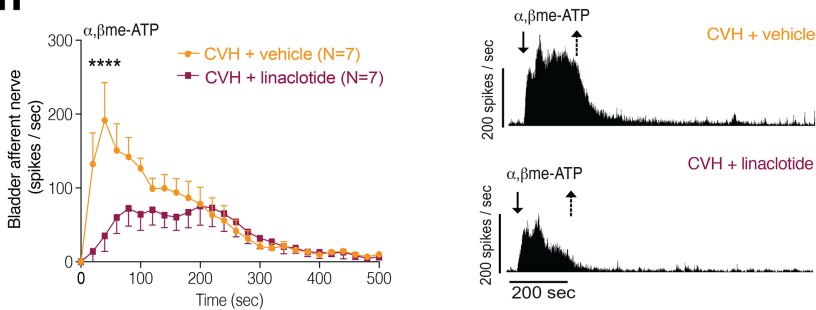

arme-ATP

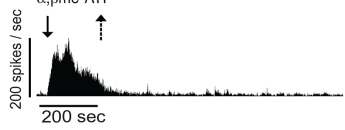

I
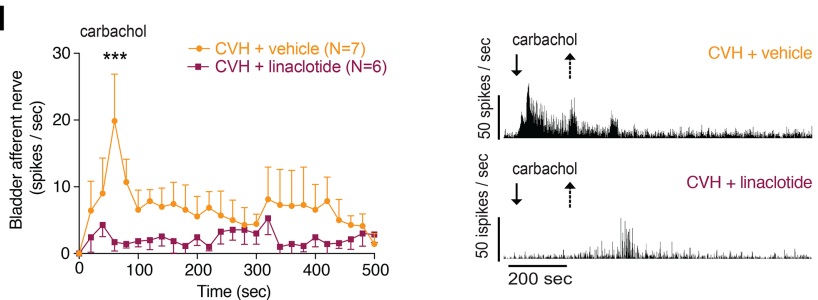

J

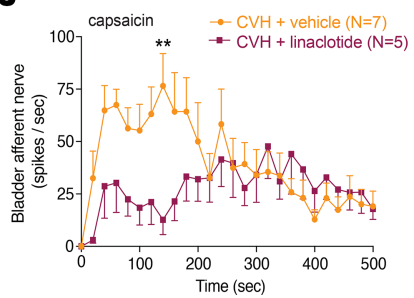

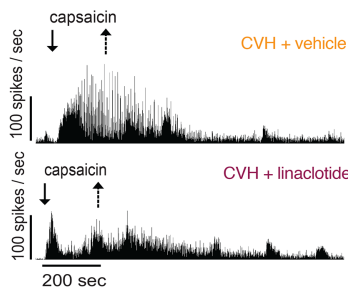

Figure 7. CVH mice display abnormal bladder afferent responses to mechanical and chemical stimuli that are reversed by chronic oral administration of linaclotide. (A) Ex vivo bladder afferent firing in response to ramp distension is increased in CVH $(N=11)$ compared with control $(N=10)$ mice $(* * * * P<$ 0.0001). (B) Ex vivo bladder multiunit recording from control or CVH mice showing action potential firing during bladder distension with intravesical saline. (C) CVH mice chronically administered linaclotide $(N=7)$ show reduced bladder afferent responses to distension compared with vehicle-treated $\mathrm{CVH}$ mice $(\mathrm{N}$ $\left.=11 ;{ }^{* *} P<0.001\right)$. (D) Ex vivo bladder multiunit recording from CVH mice treated with vehicle or daily linaclotide showing action potential firing during bladder distension with intravesical saline. (E-G) Bladder afferent responses to bath application of (E) $\alpha \beta$ Me-ATP $\left(30 \mu \mathrm{M},{ }^{* * *} P<0.001\right)$, (F) carbachol $\left(1 \mu \mathrm{M}\right.$; ${ }^{*} P<$ 0.05), and (C) capsaicin $\left(10 \mu \mathrm{M},{ }^{* * *} P<0.001\right)$ are all enhanced in CVH ( $N=5-7$ mice/group) compared with control ( $N=6$ mice/group). (H-J) Bladder afferent responses to bath application of $(\mathbf{H}) \alpha \beta$ Me-ATP $\left({ }^{* *} P<0.001\right)$, (I) carbachol $\left({ }^{* *} P<0.001\right)$, and (J) capsaicin $\left({ }^{* * *} P<0.001\right)$ are all attenuated in CVH mice chronically administered linaclotide ( $N=5-7$ mice per group) compared with vehicle-treated CVH mice $(N=7$ mice per group). Data represent mean \pm SEM. $P$ values above are based on 2-way ANOVA (A, C, and E-J), with subsequent Bonferroni post hoc test significance at individual data points indicated on E-J.

\section{Discussion}

IBS patients represent approximately $11 \%$ of the global population and suffer from chronic abdominal pain and altered gastrointestinal motility. The underlying pathogenesis of IBS is complex; however, there is a strong correlation between a prior exposure of the patient to gut infection and symptom occurrence $(1,2)$. Such bouts of acute gastroenteritis can trigger IBS symptoms in patients that persist for at least 8 years after the initial infection (29). IBS patients report visceral hypersensitivity but do not present with concurrent signs of gastrointestinal inflammation $(1,2,10)$. It is therefore suggested that patients develop neuroplasticity of visceral sensory pathways, which drives chronic abdominal pain in the absence of pathology in the postinflammatory state $(1,10)$. Here, we demonstrate that, following resolution of colitis, in the postinflammatory state, colonic nociceptors display mechanical hypersensitivity and reduced activation thresholds, 

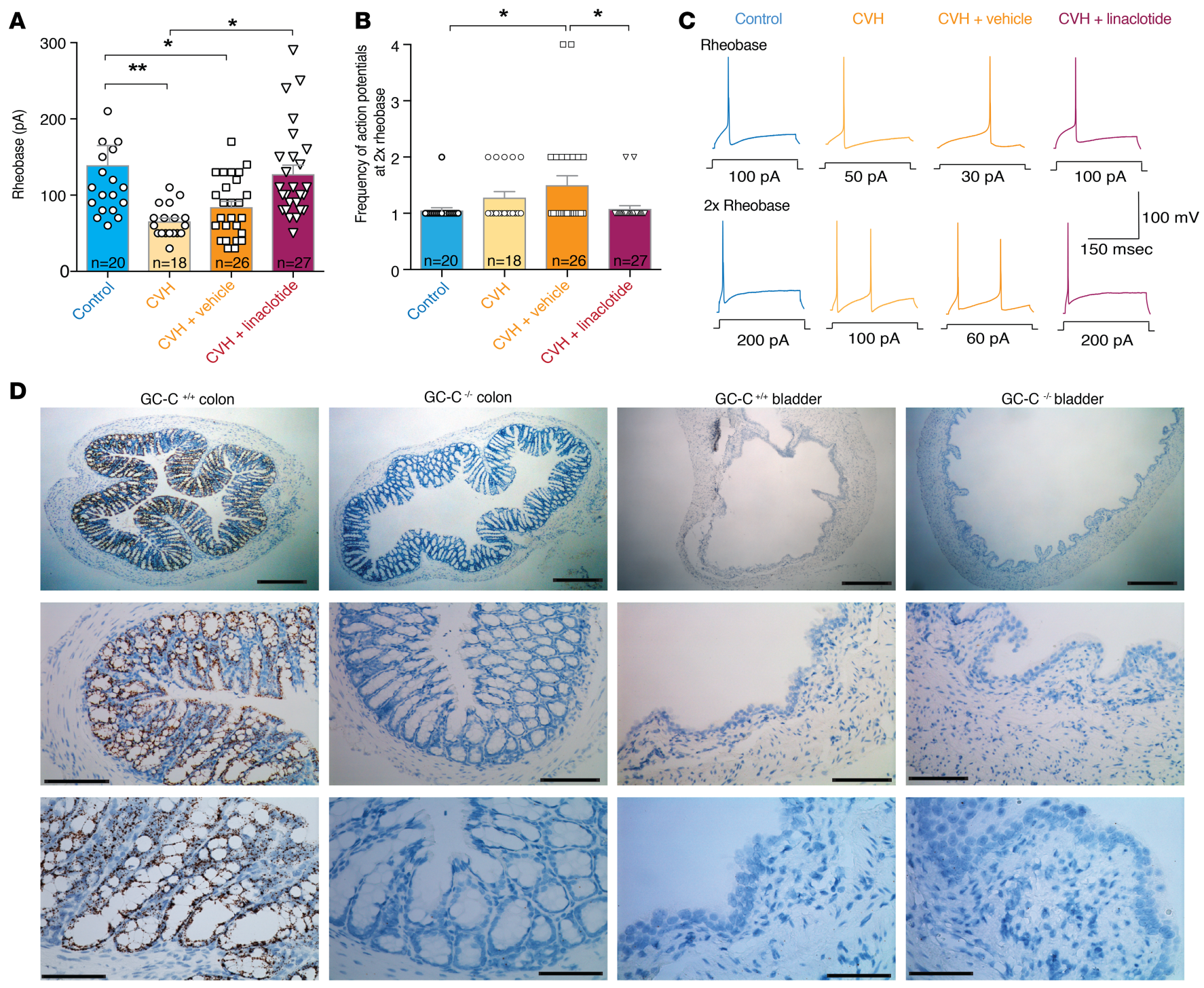

$\mathbf{E}$

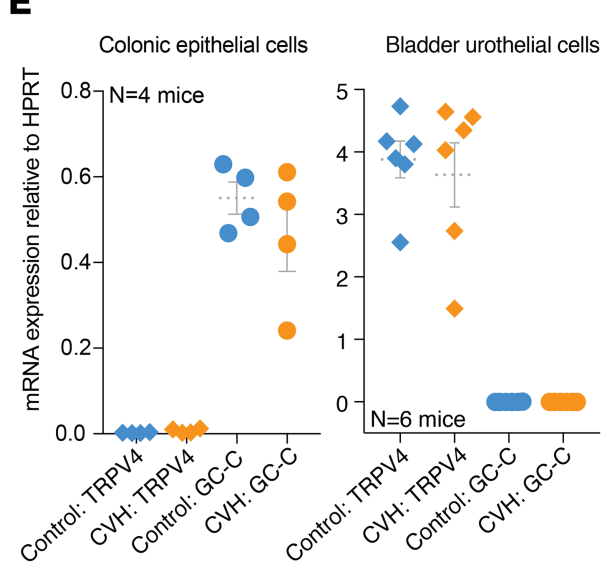

$\mathbf{F}$

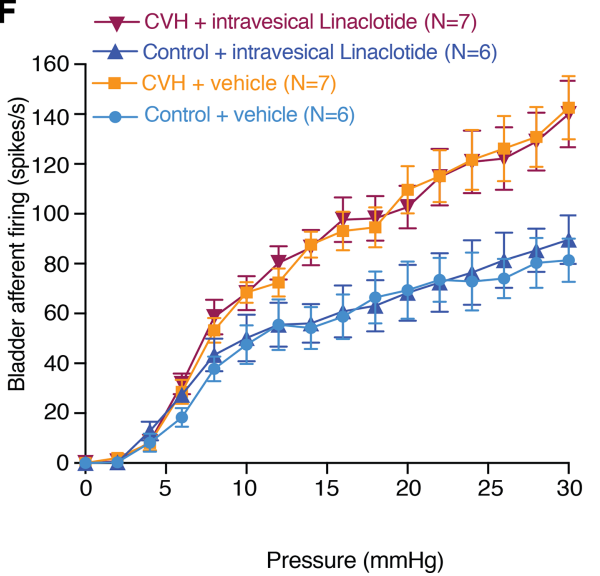

G Control

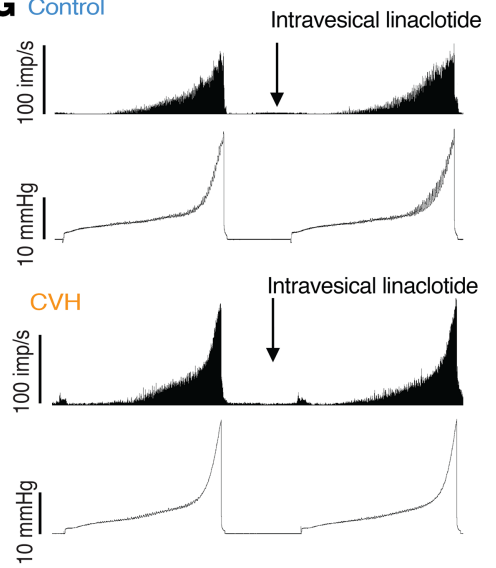

Figure 8. Chronic oral administration of linaclotide reverses CVH-induced bladder DRG neuronal hyperexcitability. (A) Whole-cell current-clamp recordings of retrogradely traced lumbosacral (LS) bladder-innervating DRG neurons, showing reduced rheobase of neurons from CVH ( $n=18$, ${ }^{*} P<$ $0.01)$ and vehicle-treated CVH $\left(n=26,{ }^{*} P<0.05\right)$ versus control $(n=20)$ mice. Bladder-innervating DRG neurons from mice chronically administered linaclotide $(n=27)$ showed normalized rheobase, which are significantly different compared to CVH neurons $\left({ }^{*} P<0.05\right)$. (B) Action potential firing at $2 \times$ rheobase is increased in vehicle-treated $\mathrm{CVH}$ mice $\left({ }^{*} P<0.05\right)$, while $\mathrm{CVH}$ mice receiving chronic linaclotide show normalized firing at $2 \times$ rheobase $\left({ }^{*} P<0.05\right)$. (C) Whole-cell current-clamp traces at rheobase and $2 \times$ rheobase in bladder-innervating neurons from control, $\mathrm{CVH}$, vehicle-treated $\mathrm{CVH}$, and linaclotide-treated CVH mice. (D) Sections of colon and bladder at increasing magnifications showing in situ hybridization. GC-C is observed in 
colonic mucosa of GC-C ( gucy $2 \mathrm{c}^{+/+}$) wild-type, but not gucy2c-null (gucy $2 \mathrm{c}^{-{ }^{-}}$) mutant mice. GC-C expression is absent in bladder samples from both gucy $2 c^{+/+}$and gucy $2 c^{-1-}$ mice. Results were repeated in $N=3$ mice/group. Scale bars: $200 \mu \mathrm{m}$ (top); $50 \mu \mathrm{m}$ (middle); $25 \mu \mathrm{m}$ (bottom). (E) Quantitative RT-PCR confirming expression of gucy2c in primary colonic epithelium and absence in primary bladder urothelium. In comparison, trpv4 is highly expressed in bladder urothelium ( $N=4-6$ mice/group). (F) Ex vivo bladder afferent mechanosensitivity to ramp distension is increased in CVH ( $N=7$ ) compared with control $(N=6)$ mice. Bladder afferent responses from control or CVH mice are unaltered following ex vivo intravesical infusion of linaclotide $(1,000 \mathrm{nM})$, showing linaclotide does not directly inhibit bladder afferents. (G) Action potential firing and intravesical pressure from control mice during bladder distension $(0-30 \mathrm{mmHg})$ with intravesical saline and linaclotide $(1,000 \mathrm{nM})$. Data represent mean $\pm \mathrm{SEM}$. $P$ values are based on 1-way ANOVA (A and $\mathbf{B}$ ) or 2-way ANOVA (F) with Bonferroni post hoc tests.

with the cell bodies of these afferents showing pronounced hyperexcitability. This increased nociceptive signal leads to increased activation of dorsal horn neurons within the spinal cord in response to noxious CRD. Notably, the neurochemistry of these activated dorsal horn neurons changes, with the recruitment of additional neuronal populations not seen in the healthy state. This may be a potential consequence of the increased density of the colonic afferent central terminals within dorsal horn lamina I and II. Correspondingly, $\mathrm{CVH}$ mice displayed allodynia and hyperalgesia in response to $\mathrm{CRD}$, indicative of chronic abdominal pain (Figure 9K). As this neuroplasticity occurs at every level of the sensory pathways innervating the colon, these changes provide an underlying mechanistic basis for the presentation of chronic abdominal pain in the absence of pathology in patients with IBS.

Having demonstrated the underlying mechanistic processes by which chronic abdominal pain can occur, we determined whether it could be reversed. Here, we show that chronic daily treatment with linaclotide, an FDA-approved GC-C agonist for the treatment of IBS-C, (a) reversed mechanical hypersensitivity of colonic nociceptors, (b) reduced the number of activated dorsal horn neurons in response to $C R D$, (c) reduced the severity of colonic afferent central terminal sprouting, and, correspondingly, (d) normalized abdominal pain to CRD back to control levels (Figure 9L). These antinociceptive and analgesic effects are driven by activation of GC-C on colonic epithelial cells and the corresponding release of $\operatorname{cGMP}(4,6,8)$. We show that linaclotide itself does not inhibit the excitability of colonic nociceptors, whereas its downstream effector cGMP causes dose-dependent inhibition of action potential firing, particularly in CVH states. We also show conclusively for the first time to our knowledge that cGMP acts on a membrane target that is accessed from an extracellular site to inhibit nociceptors. The precise molecular target that cGMP acts on extracellularly remains elusive but is the subject of continued investigation. We also observed that human DRG neurons, with a pathological phenotype following culture in inflammatory mediators, have an increased responsiveness to cGMP. Taken together, these findings demonstrate how chronic daily linaclotide treatment reduces chronic colonic hypersensitivity and chronic abdominal pain in a preclinical model of IBS. This may explain why long-term clinical use of linaclotide results in more IBS-C patients reporting abdominal pain relief over time $(4,5)$, particularly if their underlying neuroplasticity is more entrenched following years of symptoms.

Recently, there has been an increased appreciation that IBS patients also suffer from a wide-range of extraintestinal comorbidities, including OAB and IC-PBS (13). Although these symptoms contribute to the poor quality of life experienced by IBS patients, the underlying etiology of these comorbidities has been unclear. Here, we show that colitis-induced chronic colonic afferent hypersensitivity subsequently results in chronic bladder afferent hypersensitivity to mechanical and chemical stimuli. This leads to chronic hyperexcitability of bladder-innervating DRG neurons and, ultimately, chronic dysfunction of bladder voiding (Figure 9K). These chronic changes in bladder function are not due to colitis-induced inflammation of the bladder, nor due to changes in the contractility of the bladder. We show that bladder dysfunction takes several weeks to develop and occurs following the resolution of colitis and following the development of colonic hypersensitivity, which commonly presents 3-7 days after induction of colitis (20,30). Accordingly, these findings provide, for the first time to our knowledge, a mechanistic basis as to why IBS patients may also suffer from concurrent OAB/IC-PBS, particularly without a prior bladder infection or insult.

Our dual-retrograde tracing studies from the colon and bladder identified a small population of colon-innervating DRG neurons that also innervate the bladder ( $15 \%)$, called dichotomizing afferents. As previously reported, these afferents have a single soma in the DRG but bifurcating axons that project to either the colon or bladder, respectively (14-16). Accordingly, dichotomizing afferents have been proposed as a mechanism for viscero-visceral crosstalk and the basis of cross-organ sensitization $(15,16,31,32)$. These dichotomizing afferents likely contribute directly to the cross-organ sensitization observed from the colon to the bladder in the current study, as they provide a clear mechanism by which sensitization of colonic afferents results in sensitization of bladder afferents. However, as they represent only approximately $15 \%$ of colon-innervating afferents, they are 
A

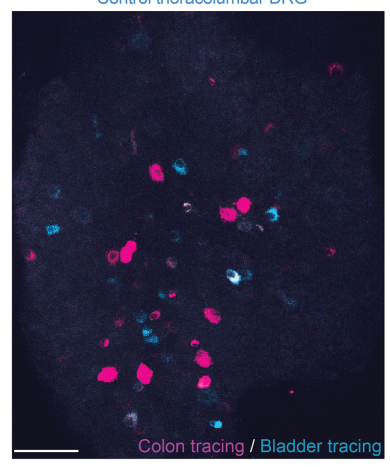

B

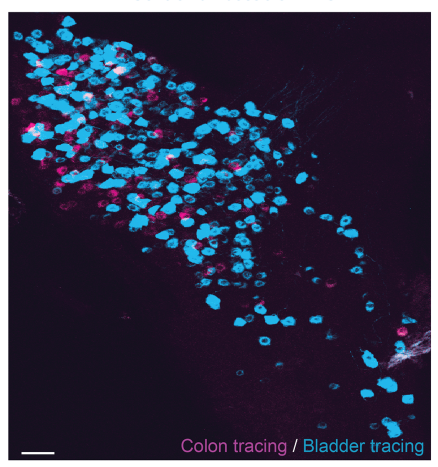

G

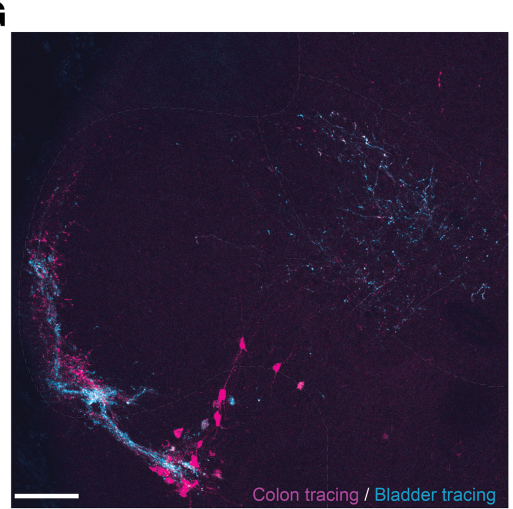

K

Chronic Visceral Hypersensitivity State

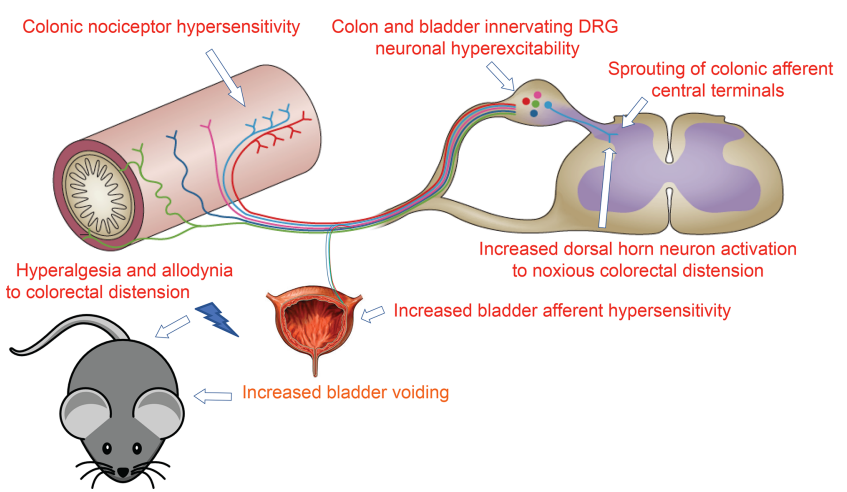

C
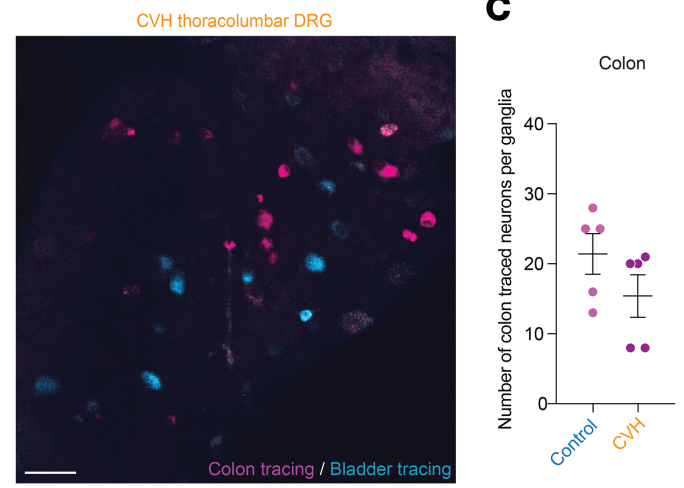

D

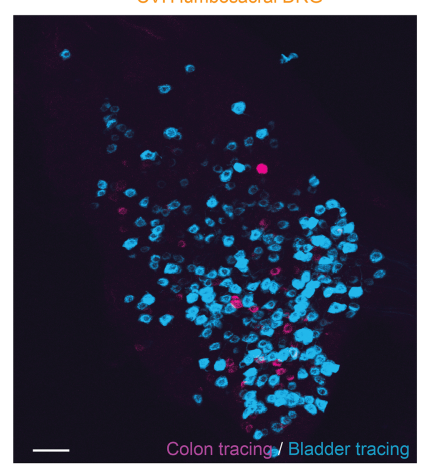

$\mathbf{H}$

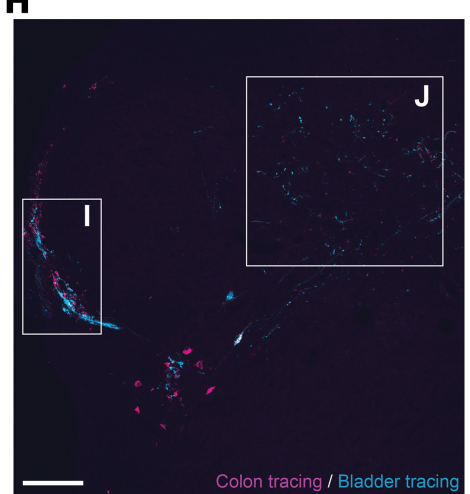

L1 DRG
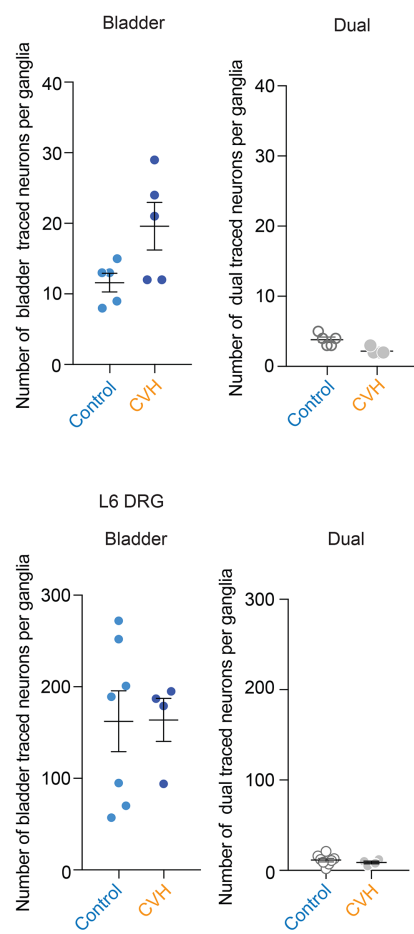

I

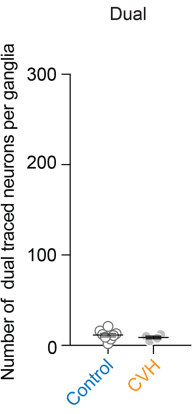

J
F

E Dual as a $\%$ of colon

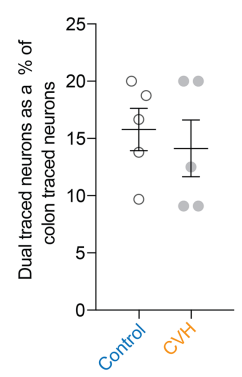

6 DRG

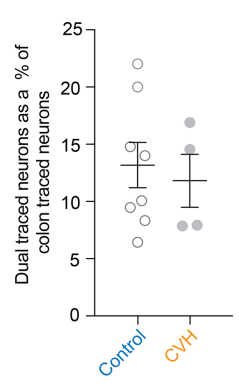

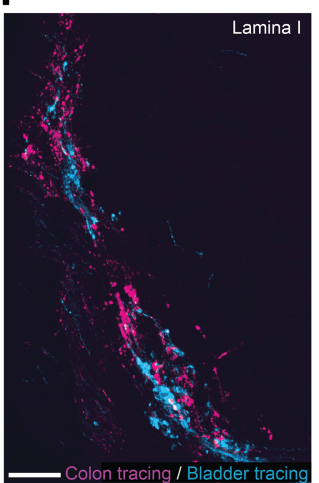

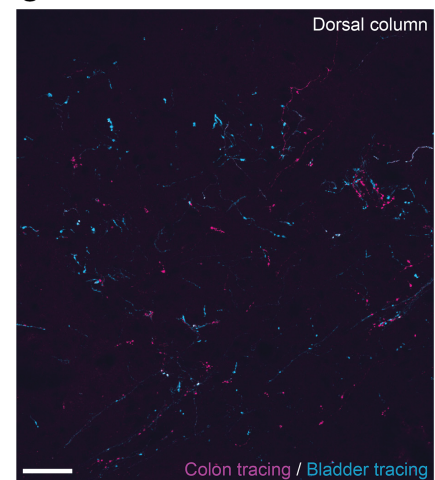

$\mathbf{L}$

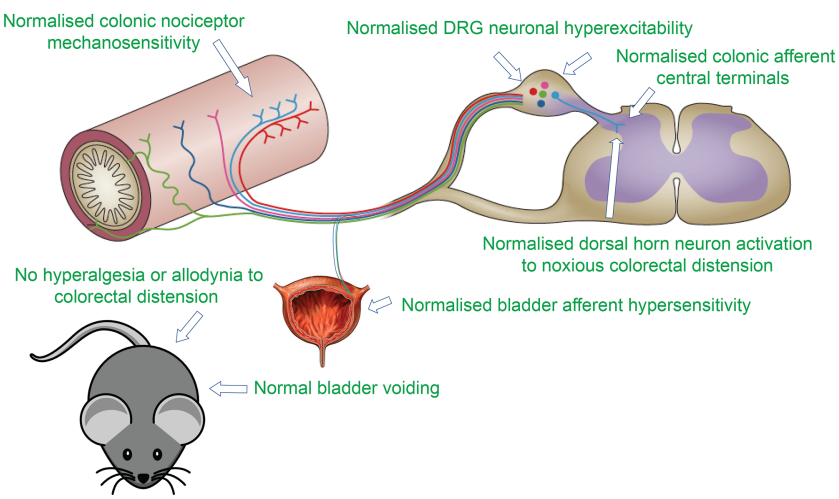

Figure 9. Colon- and bladder-innervating dorsal root ganglion neurons are largely distinct but their central terminals are closely apposed within the spinal cord. Retrograde tracer from colon- (CTB-555; magenta), bladder- (CTB-488; blue), or dual-traced (white) neurons in CLARITY cleared TL (L1) and LS (L6) dorsal root ganglia (DRG) from (A) control and (B) CVH mice. Scale bar: $100 \mu \mathrm{m}$. (C and D) Quantitative data of the number of colon-only, bladder-only, or dual-traced neurons per ganglia within (C) L1 and (D) L6 DRG ( $N=3-5$ mice per group). Data indicate no significant difference in the total number of colon-only, bladder-only ( $P=0.058$, unpaired $t$ test), or dual-traced populations of neurons in control and CVH states. (E and $\mathbf{F}$ ) Quantitative data of dual-traced neurons, 
expressed as a percentage of colon-innervating neurons in (E) L1 and (F) L6 DRG. Data indicate no significant difference between control and CVH. (G and $\mathbf{H})$ Spinal cord (50- $\mu \mathrm{m}$ sections) showing retrograde tracer from the colon (magenta) or bladder (blue). Imaging reveals that the central terminals of colon-innervating or bladder-innervating DRG neurons are in close apposition to one another within the dorsal horn, including within the lateral spinal nucleus and the lumbar dorsal column (repeated in $N=3$ mice). Scale bar: $100 \mu \mathrm{m}$. (I and J) Enlarged images from insets within H. Scale bar: $30 \mu \mathrm{m}$. (K) Diagrams detailing changes in colon- and bladder-innervating sensory pathways during CVH and (L) how chronic linaclotide treatment normalizes colonic nociception and, subsequently, normalizes bladder afferent function and voiding. Data represent mean \pm SEM. $P$ values are based on unpaired $t$ tests (C-F).

unlikely to represent the entire mechanistic story of how transient colitis induces persistent bladder hypersensitivity. This is particularly apparent from our bladder ex vivo and patch-clamp recordings, whereby the vast majority of bladder-innervating afferents/neurons from CVH mice display hypersensitivity or hyperexcitability.

Interestingly, our retrograde tracing studies identified that the cell soma of distinct colon- and bladder-innervating afferents lie in close proximity to one another within the DRG. This is an important finding, as recent studies have shown that coupled activation of DRG neurons contributes to chronic pain (33). In such a scenario, and following inflammation or nerve injury, adjacent neurons within the DRG activate together, and this is mediated by an injury-induced upregulation of gap junctions in the glial cells surrounding DRG neurons (33). Interestingly, such interactions rarely occur in naive animals (33). Such a mechanism provides a second plausible explanation whereby hypersensitive colon-innervating DRG neurons can subsequently sensitize bladder-innervating DRG neurons.

A third potential site of interaction is within the dorsal horn of the spinal cord. In the current study, we show that the central terminals of colon-innervating and bladder-innervating afferents are located in close proximity to one another within the spinal cord, thus projecting into shared dorsal horn circuits. In CVH mice, we observed an increased density and distribution of the central terminals of colonic afferents within the dorsal horn and activation of a different population of dorsal horn neurons in response to noxious CRD. Interestingly, a greater number of dorsal horn neurons activated in response to noxious CRD were immunoreactive for GABA, traditionally thought to identify inhibitory interneurons (22). These findings potentially suggest that inhibition of inhibitory pathways may contribute to the neuroplasticity underling cross-organ sensitization between the colon and bladder. Therefore, colitis-induced enhancement of activation of spinal cord circuits could alter the spinal cord processing of bladder afferent input and therefore the sensory input then relayed into central micturition control regions. Such colitis-evoked changes could occur via the activation of spinal microglia, which are driving forces for maintaining pathological pain and for visceral and somatic sensitization following peripheral inflammation $(34,35)$. Activated microglia release neuroexcitatory substances, proinflammatory cytokines, and growth factors, potentiating ectopic activation of dorsal horn neurons and neurite sprouting. Accordingly, the precise mechanisms by which sensitization of colonic afferent pathways results in persistent bladder afferent dysfunction is the subject of ongoing investigation.

Having established that chronic bladder dysfunction occurs in CVH states, we determined, in the first study of its kind to our knowledge, that daily linaclotide treatment is able to normalize bladder-voiding patterns and attenuate neuronal and mechanical bladder hypersensitivity of mice with CVH (Figure 9L). This also includes normalization of enhanced responses to TRPV1, P2X $\mathrm{X}_{3}$, and muscarinic receptor agonists in $\mathrm{CVH}$ states, targets that are known to be essential for normal bladder function (36-38). We observed that it takes 2 weeks of linaclotide treatment for the inhibitory actions on bladder afferents to become clearly evident. Linaclotide has a low oral bioavailability and remains localized to the colon $(7,8)$. We also showed that GC-C is not expressed by bladder urothelial cells and that application of linaclotide into the lumen of the bladder does not influence mechanosensation or muscle compliance. Taken together, these findings indicate that normalization of bladder function by daily linaclotide treatment occurs via an indirect inhibition of bladder afferent signaling. We hypothesize that this could occur via (a) reduced nociceptive signaling from the colon, resulting in reduced neuronal firing of dichotomizing bladder afferents; or (b) via decreased coupled activation of colon-only and bladder-only innervating neurons within the DRG; or, finally, (c) linaclotide-induced reversal of neuroplasticity within the dorsal horn, restoring the circuitry for normal bladder function.

Overall, we have identified that both chronic abdominal pain and chronic bladder dysfunction occur in CVH states following the resolution of colitis. Our findings shed light on the mechanisms of cross-organ sensitization and provide the tantalizing prospect of treating multiple syndromes concurrently, while avoiding the debilitating side effects of current opioid-based pain treatments. Our findings may also have 
relevance beyond patients with IBS, as $30 \%$ of patients primarily defined as having OAB or IC-PBS exhibit symptoms of IBS among their most common comorbidities (11). Linaclotide is FDA approved, well tolerated by patients, with few side effects, and may therefore provide a safe alternative treatment for these patients. Consequently, our findings identify mechanisms that may be taken advantage of in the future for the treatment of comorbid pelvic pain symptoms.

\section{Methods}

For extensive and details descriptions of the methodology used, please see the Supplemental Methods.

Animals and ethics. Male C57BL/6J mice aged 13-17 weeks were used for adult studies. Some experiments also utilized male mice lacking GC-C (gucy $2 \mathrm{C}^{\prime-}$, in-house breeding colony at SAHMRI), which were generated as described previously (39). For FRET studies, R26-CAG-mcGi500 mice, which contain the sensor cGi500 under control of the ubiquitous CAG promoter, were used. R26-CAG-mcGi500 mice were generated as described previously (27).

Mouse model of $\mathrm{CVH}$. Mice were administered intracolonic TNBS and developed colitis $(4,18-21)$, with significant increases in MPO activity (Supplemental Figure 1). While colonic inflammation spontaneously healed over a 7-day period (Supplemental Figure 1), these mice subsequently developed chronic colonic afferent hypersensitivity in the postinflammatory state (4, 18-21).

Study design. Mice were randomly assigned to either (a) control or (b) CVH cohorts and then randomly assigned to 1 of 3 groups: those that received (a) no gavage, or (b) once daily 100- $\mu$ l oral gavage of linaclotide ( $3 \mu \mathrm{g} / \mathrm{kg} / \mathrm{d}$ ) for 14 days, or (c) vehicle (water) once daily $100-\mu 1$ oral gavage for 14 days. Daily oral administration began from 14 days after TNBS administration (or time/day matched for control animals) until 28 days after TNBS administration.

In vivo VMR to CRD. We assessed in vivo visceral sensitivity to CRD (the distension pressures 20, 40, 60 , and $80 \mathrm{mmHg}$ were used for these studies, each 20 -second duration, applied at 4-minute intervals) using abdominal electromyography in fully awake animals. Colonic compliance was assessed by applying graded volumes (40-200 $\mu 1,20$-second duration) (40-42).

Ex vivo single-fiber colonic nociceptor recordings. Ex vivo single-fiber colonic nociceptor recordings were

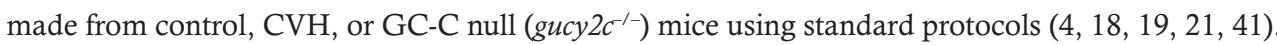

Visualization of $p E R K$-activated neurons within the dorsal horn of the spinal cord following noxious CRD. 80 mmHg CRD was performed (10 seconds on, 5 second deflation, repeated 5 times). After anesthetic overdose, mice were fixed by transcardial perfusion, the spinal cord removed and cryoprotected. Frozen sections were cut and incubated with monoclonal-rabbit anti-phosphorylated-MAP-kinase-ERK-1/2 (pERK) (Cell Signaling Technology, 4370) visualized with Alexa Fluor 488 (ThermoFisher Scientific, A-21441) (4, 23).

Retrograde tracing to label the cell bodies or central terminals of colon or bladder afferents. Cholera toxin subunit-B conjugated to Alexa Fluor 555 (CTB-555) was injected at 3 sites subserosally within the distal colon, while CTB-488 was injected into 3 sites into the bladder. Animals were left to recover for 4 days to identify cell bodies within the DRG or for 7 days to identify central terminals within the spinal cord $(18,23,41)$.

Patch-clamp recordings of colon- or bladder-innervating DRG neurons. Whole-cell patch-clamp recordings in current clamp mode were made from retrogradely traced colon- or bladder- innervating DRG neurons. 10 pA-depolarizing pulses of 500-millisecond duration were applied to determine rheobase (current required to fire an action potential) $(18,21)$.

Human DRG neuron patch-clamp recordings. Human DRG neurons were acquired from organ donors with ethical consent, and whole-cell patch-clamp recordings were performed in current-clamp mode to determine rheobase (18).

FRET-based cGMP imaging in DRG neurons. DRG neurons were isolated from R26-CAG-mcGi500(L1) embryos that express a membrane-targeted version of the FRET-based cGMP sensor cGi500 under control of the ubiquitous CAG promoter. FRET/cGMP imaging was performed 24 hours after plating DRG neurons (27).

Bladder-voiding pattern analysis. Mice were individually housed in cages lined with filter paper for 3 hours. Filter paper was collected immediately prior to intracolonic TNBS administration and at days 7 , 14, 21, and 28 after TNBS administration or the same time point for control mice. Filter paper was imaged using a Bio-Rad ultraviolet transilluminator and digitized into binary images using ImageJ (NIH).

Ex vivo electrophysiology for bladder afferent recordings and contractility studies. Whole pelvic nerve recordings were conducted in response to ramp distension $(30 \mu 1 / \mathrm{min}$ to $30 \mathrm{mmHg})(37,43,44)$. Studies 
also determined the responsiveness of bladder afferents to intravesically applied linaclotide or $\alpha \beta \mathrm{Me}$ ATP, carbachol, or capsaicin.

In situ hybridization $m R N A$ detection of GC-C. In situ hybridization mRNA detection of GC-C was performed in formalin-fixed, paraffin-embedded bladder and colon sections from $\mathrm{GC}_{-} \mathrm{C}^{+/+}$and $\mathrm{GC}-\mathrm{C}^{-/-}$mice using the RNAscope 2.5 LS assay.

Q-RT-PCR for GC-C in bladder and colonic epithelial cells. The urothelial/epithelial layers were removed from bladder or colon, respectively, isolated and RNA extracted. Q-RT-PCR was performed with commercially available hydrolysis TaqMan probes (Thermo Fisher Scientific) for GC-C, TRPV4, and Hprt. Relative abundance was estimated using $\Delta \mathrm{Cq}$ method.

Statistics. Data are expressed as mean \pm SEM or the percentage of neurons/afferents. Figures were prepared in GraphPad Prism 7 Software; $N$ equals the number of animals, while $n$ equals the number of neurons/ afferents. Differences were considered significant at a level of $P<0.05$. Visceromotor responses (VMRs) to CRD data were statistically analyzed by generalized estimating equations followed by LSD post hoc test using SPSS 23.0 (IBM). All other data were analyzed using GraphPad Prism 7. These data were analyzed using (a) 1-way ANOVA. If data were normally distributed, they were analyzed using repeated-measures 1-way ANOVA, with post hoc analysis conducted by making all possible comparisons among the treatment groups with Tukey's tests. If data were nonparametric, they were analyzed using Kruskal-Wallis 1-way ANOVA, and Dunn's test was used to assess any post hoc differences in comparisons among all groups. (b) If data were normally distributed, they were analyzed by repeated-measures 2-way ANOVA with post hoc Bonferroni or Tukey's tests and (c) paired or unpaired 2-tailed t tests or (d) $\chi^{2}$ analysis. The specific tests used to analyze each data set are indicated within the individual figure legends.

Study approval. The animal ethics committees of SAHMRI, Flinders University, the University of Adelaide, and the University of Tübingen approved experiments involving animals. All experiments conformed to the relevant regulatory standards and the ARRIVE guidelines. All human tissues used for the study were obtained by legal consent from organ donors in the US. AnaBios's procurement network includes only US-based organ procurement organizations and hospitals. Policies for donor screening and consent are the ones established by the United Network for Organ Sharing. Organizations supplying human tissues to AnaBios follow the standards and procedures established by the US Centers for Disease Control and are inspected biannually by the Department of Health and Human Services. Tissue distribution is governed by internal IRB procedures and compliance with HIPAA regulations regarding patient privacy. All transfers of donor organs to AnaBios are fully traceable and periodically reviewed by US federal authorities.

\section{Author contributions}

$\mathrm{AD}, \mathrm{JC}, \mathrm{JM}, \mathrm{CBK}$, ISS, and SMB designed, performed, and analyzed the VMR to CRD studies. JC and SMB designed, performed, and analyzed the colonic afferent recordings. AMH and SMB designed, performed, and analyzed the pERK and retrograde tracing studies. SP, RF, CBK, and ISS designed, performed, and analyzed the FRET cGMP experiments. LG, GYR, and SMB designed, performed, and analyzed the patch-clamp experiments on mouse colon- and bladder-innervating DRG neurons. LG and SMB designed, performed, and analyzed the bladder afferent recordings and bladder-voiding patterns analysis. PM, AG, ISS, and SMB designed, performed, and analyzed the patch-clamp recordings on human DRG neurons. PG and GH designed, performed, and analyzed the in situ hybridization studies. SGC and SMB designed, performed, and analyzed the Q-RT-PCR and MPO experiments. AMH and SGC performed DRG CLARITY experiments. LG and SGC performed the histology experiments. All authors contributed to the discussion and interpretation of the results. SMB wrote the manuscript with contributions and suggestions from all authors.

\section{Acknowledgments}

We thank Tracey O'Donnell and Jessi Moore for their technical assistance. This work was supported by research grant funding from Ironwood Pharmaceuticals and Allergan (to SMB), DFG grant FOR 2060 (FE 438/5-2 and FE 438/6-2 to RF), the Fund for Science (to RF), a National Health and Medical Research Council of Australia (NHMRC) R.D. Wright Biomedical Research Fellowship (APP1126378 to SMB), NHMRC Australia project grants (1083480, 1139366, and 1140297 to SMB), an Australian Research Council (ARC) Discovery Early Career Research Award (DE130100223 to AMH), and an ARC Discovery Project grant (DP180101395 to AMH and SMB). 
Address correspondence to: Stuart M. Brierley, Visceral Pain Research Group, Level 7, South Australian Health and Medical Research Institute (SAHMRI), North Terrace, Adelaide, SA 5000, Australia. Phone: 61.8.8128.4848; Email: stuart.brierley@flinders.edu.au.

CBK's present address is: Synlogic, Cambridge, Massachusetts, USA.

ISS's present address is: Decibel Therapeutics, Cambridge, Massachusetts, USA.

1. Enck P, et al. Irritable bowel syndrome. Nat Rev Dis Primers. 2016;2:16014.

2. Chey WD, Kurlander J, Eswaran S. Irritable bowel syndrome: a clinical review. JAMA. 2015;313(9):949-958.

3. Volkow ND, McLellan AT. Opioid abuse in chronic pain--misconceptions and mitigation strategies. N Engl J Med. 2016;374(13):1253-1263.

4. Castro J, et al. Linaclotide inhibits colonic nociceptors and relieves abdominal pain via guanylate cyclase-C and extracellular cyclic guanosine 3',5'-monophosphate. Gastroenterology. 2013;145(6):1334-46.e1.

5. Chey WD, et al. Linaclotide for irritable bowel syndrome with constipation: a 26-week, randomized, double-blind, placebo-controlled trial to evaluate efficacy and safety. Am J Gastroenterol. 2012;107(11):1702-1712.

6. Bryant AP, et al. Linaclotide is a potent and selective guanylate cyclase $\mathrm{C}$ agonist that elicits pharmacological effects locally in the gastrointestinal tract. Life Sci. 2010;86(19-20):760-765.

7. Busby RW, et al. Pharmacologic properties, metabolism, and disposition of linaclotide, a novel therapeutic peptide approved for the treatment of irritable bowel syndrome with constipation and chronic idiopathic constipation. J Pharmacol Exp Ther. 2013;344(1):196-206.

8. Busby RW, et al. Linaclotide, through activation of guanylate cyclase C, acts locally in the gastrointestinal tract to elicit enhanced intestinal secretion and transit. Eur J Pharmacol. 2010;649(1-3):328-335.

9. Eutamene H, et al. Guanylate cyclase C-mediated antinociceptive effects of linaclotide in rodent models of visceral pain. Neurogastroenterol Motil. 2010;22(3):312-e84.

10. Brierley SM, Linden DR. Neuroplasticity and dysfunction after gastrointestinal inflammation. Nat Rev Gastroenterol Hepatol. 2014;11(10):611-627.

11. Grundy L, Brierley SM. Cross-organ sensitization between the colon and bladder: to pee or not to pee? Am J Physiol Gastrointest Liver Physiol. 2018;314(3):G301-G308.

12. Whitehead WE, Palsson O, Jones KR. Systematic review of the comorbidity of irritable bowel syndrome with other disorders: what are the causes and implications? Gastroenterology. 2002;122(4):1140-1156.

13. Cukier JM, Cortina-Borja M, Brading AF. A case-control study to examine any association between idiopathic detrusor instability and gastrointestinal tract disorder, and between irritable bowel syndrome and urinary tract disorder. Br J Urol. 1997;79(6):865-878.

14. Lei Q, Malykhina AP. Colonic inflammation up-regulates voltage-gated sodium channels in bladder sensory neurons via activation of peripheral transient potential vanilloid 1 receptors. Neurogastroenterol Motil. 2012;24(6):575-585.

15. Lei Q, et al. Lack of transient receptor potential vanilloid 1 channel modulates the development of neurogenic bladder dysfunction induced by cross-sensitization in afferent pathways. J Neuroinflammation. 2013;10:3.

16. Malykhina AP, Qin C, Greenwood-van Meerveld B, Foreman RD, Lupu F, Akbarali HI. Hyperexcitability of convergent colon and bladder dorsal root ganglion neurons after colonic inflammation: mechanism for pelvic organ cross-talk. Neurogastroenterol Motil. 2006;18(10):936-948.

17. Xia CM, et al. Up-regulation of brain-derived neurotrophic factor in primary afferent pathway regulates colon-to-bladder cross-sensitization in rat. J Neuroinflammation. 2012;9:30.

18. Castro J, et al. $\alpha$-Conotoxin Vc1.1 inhibits human dorsal root ganglion neuroexcitability and mouse colonic nociception via GABAB receptors. Gut. 2017;66(6):1083-1094.

19. de Araujo AD, et al. Selenoether oxytocin analogues have analgesic properties in a mouse model of chronic abdominal pain. Nat Commun. 2014;5:3165.

20. Hughes PA, Brierley SM, Martin CM, Brookes SJ, Linden DR, Blackshaw LA. Post-inflammatory colonic afferent sensitisation: different subtypes, different pathways and different time courses. Gut. 2009;58(10):1333-1341.

21. Osteen JD, et al. Selective spider toxins reveal a role for the Nav1.1 channel in mechanical pain. Nature. 2016;534(7608):494-499.

22. Todd AJ. Identifying functional populations among the interneurons in laminae I-III of the spinal dorsal horn. Mol Pain. 2017;13:1744806917693003

23. Harrington AM, Brierley SM, Isaacs N, Hughes PA, Castro J, Blackshaw LA. Sprouting of colonic afferent central terminals and increased spinal mitogen-activated protein kinase expression in a mouse model of chronic visceral hypersensitivity. J Comp Neurol. 2012;520(10):2241-2255.

24. Werner K, Schwede F, Genieser HG, Geiger J, Butt E. Quantification of cAMP and cGMP analogs in intact cells: pitfalls in enzyme immunoassays for cyclic nucleotides. Naunyn Schmiedebergs Arch Pharmacol. 2011;384(2):169-176.

25. Russwurm M, Mullershausen F, Friebe A, Jäger R, Russwurm C, Koesling D. Design of fluorescence resonance energy transfer (FRET)-based cGMP indicators: a systematic approach. Biochem J. 2007;407(1):69-77.

26. Thunemann M, Fomin N, Krawutschke C, Russwurm M, Feil R. Visualization of cGMP with cGi biosensors. Methods Mol Biol. 2013;1020:89-120.

27. Thunemann M, et al. Transgenic mice for cGMP imaging. Circ Res. 2013;113(4):365-371.

28. Janssen DA, Hoenderop JG, Jansen KC, Kemp AW, Heesakkers JP, Schalken JA. The mechanoreceptor TRPV4 is localized in adherence junctions of the human bladder urothelium: a morphological study. J Urol. 2011;186(3):1121-1127. 
29. Marshall JK, et al. Eight year prognosis of postinfectious irritable bowel syndrome following waterborne bacterial dysentery. Gut. 2010;59(5):605-611.

30. Hughes PA, et al. Increased $\kappa$-opioid receptor expression and function during chronic visceral hypersensitivity. Gut. 2014;63(7):1199-1200.

31. Christianson JA, Liang R, Ustinova EE, Davis BM, Fraser MO, Pezzone MA. Convergence of bladder and colon sensory innervation occurs at the primary afferent level. Pain. 2007;128(3):235-243.

32. Yoshikawa S, et al. Pelvic organ cross-sensitization to enhance bladder and urethral pain behaviors in rats with experimental colitis. Neuroscience. 2015;284:422-429.

33. Kim YS, et al. Coupled activation of primary sensory neurons contributes to chronic pain. Neuron. 2016;91(5):1085-1096.

34. Basso L, et al. Granulocyte-colony-stimulating factor (G-CSF) signaling in spinal microglia drives visceral sensitization following colitis. Proc Natl Acad Sci USA. 2017;114(42):11235-11240.

35. Saab CY, Wang J, Gu C, Garner KN, Al-Chaer ED. Microglia: a newly discovered role in visceral hypersensitivity? Neuron Glia Biol. 2006;2(4):271-277.

36. Cockayne DA, et al. Urinary bladder hyporeflexia and reduced pain-related behaviour in P2X3-deficient mice. Nature. 2000;407(6807):1011-1015.

37. Daly D, Rong W, Chess-Williams R, Chapple C, Grundy D. Bladder afferent sensitivity in wild-type and TRPV1 knockout mice. J Physiol (Lond). 2007;583(Pt 2):663-674.

38. Kim Y, Yoshimura N, Masuda H, de Miguel F, Chancellor MB. Antimuscarinic agents exhibit local inhibitory effects on muscarinic receptors in bladder-afferent pathways. Urology. 2005;65(2):238-242.

39. Mann EA, Jump ML, Wu J, Yee E, Giannella RA. Mice lacking the guanylyl cyclase C receptor are resistant to STa-induced intestinal secretion. Biochem Biophys Res Commun. 1997;239(2):463-466.

40. Carstens BB, et al. Structure-activity studies of cysteine-rich $\alpha$-conotoxins that inhibit high-voltage-activated calcium channels via GABA(B) receptor activation reveal a minimal functional motif. Angew Chem Int Ed Engl. 2016;55(15):4692-4696.

41. Castro J, et al. Cyclic analogues of $\alpha$-conotoxin Vc1.1 inhibit colonic nociceptors and provide analgesia in a mouse model of chronic abdominal pain. Br J Pharmacol. 2018;175(12):2384-2398.

42. Christianson JA, Gebhart GF. Assessment of colon sensitivity by luminal distension in mice. Nat Protoc. 2007;2(10):2624-2631.

43. Daly DM, Nocchi L, Liaskos M, McKay NG, Chapple C, Grundy D. Age-related changes in afferent pathways and urothelial function in the male mouse bladder. J Physiol (Lond). 2014;592(3):537-549.

44. Grundy L, Daly DM, Chapple C, Grundy D, Chess-Williams R. TRPV1 enhances the afferent response to P2X receptor activation in the mouse urinary bladder. Sci Rep. 2018;8(1):197. 